ISSN: 1641-4713; e-ISSN: 2081-1160

DOI: https://doi.org/10.36551/2081-1160.2021.27.159-191

\title{
Análisis espacial electoral de los factores socioeconómicos entre 2009-2018 para Nuevo León, México
}

Electoral spatial analysis of socioeconomic factors between 2009-2018 for Nuevo Leon, Mexico

Sandra Edith Medellín Mendoza

Instituto para el Desarrollo Regional Escuela de Gobierno y Transformación Pública del Tecnológico de Monterrey

Nuevo León, México ORCID iD: https://orcid.org/0000-0002-9482-2675

E-mail: semedellin@tec.mx

Laura Nelly Medellín Mendoza

Facultad de Derecho y Criminología Universidad Autónoma de Nuevo León, México ORCID iD: https://orcid.org/0000-0003-1049-1635

E-mail: laura.medellinmn@uanl.edu.mx

Recepción: 3.01.2021

Aprobación: 21.06.2021

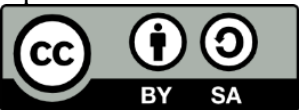

Resumen: El objetivo de esta investigación es indicar los principales determinantes socioeconómicos de la participación electoral en Nuevo León de 2009 al 2018, tomando en cuenta la distribución espacial de las variables a nivel municipal. Debido a la presencia de dependencia espacial en los datos electorales y socioeconómicos, se hace un análisis de regresión de mínimos cuadrados ordinarios donde se incluye este componente espacial. Como resultado principal del análisis de regresión espacial, se encuentra que para todos los periodos electorales analizados se da un grado de marginación alta que impacta positivamente en el nivel de participación electoral para un año determinado.

Palabras clave: participación electoral, regresión espacial, municipios, marginación, desempleo.

Abstract: The objective of this research is to indicate the main socioeconomic determinants of electoral participation in Nuevo León from 2009 to 2018, taking into account the spatial distribution 
of the variables at the municipal level. Due to the presence of spatial dependence in the electoral and socioeconomic data, a regression analysis is performed where this spatial component is modeled. As the main result of the spatial regression analysis, it is found that for all the electoral periods analyzed, a high degree of marginalization positively impacts the level of electoral participation for a given year.

Keywords: electoral participation, spatial analysis, municipalities, marginalization, unemployment.

\section{INTRODUCCIÓN}

La participación electoral de los ciudadanos para la elección de sus 51 autoridades municipales en el estado de Nuevo León en los últimos años ha registrado un comportamiento a la baja. En 2009 este indicador era un 65\%, mientras que nueve años más tarde, en 2018 esta participación llegó a ser del 63.3\%. ${ }^{1}$ De acuerdo con los datos de la Comisión Estatal Electoral de Nuevo León, el nivel más alto de participación electoral fue en 2015, cuando este indicador alcanzó el $67.7 \%$ en promedio.

Las causas por las que este desanimo surge en la participación electoral han sido estudiadas tradicionalmente, pero en la literatura no hay consenso sobre los efectos que puede tener la adversidad económica sobre la participación electoral. Si bien se ha encontrado un efecto movilizador, también se ha verificado la hipótesis del abandono; ${ }^{2}$ es decir, la adversidad económica provoca efectivamente el descenso en la participación electoral (Bartle et al., 2017; Carreras \& Castañeda-Angarita, 2019; Lavezzolo, 2008). Pero existen otros trabajos que parten de la primera ley de la Geografía de Tobler que indica que "todo depende de todo, pero las cosas que están más cerca son las que están más relacionadas que las cosas que están más distantes"; este enfoque conecta con la idea de que las personas que comparten un entorno, tienden a adoptar una conducta política similar (Losada \& Casas, 2008, p. 157).

Es por eso que este artículo se enfoca en explicar cómo el comportamiento electoral y la ubicación espacio-temporal a nivel municipal pueden estar relacionados con determinantes como la educación, la violencia, la marginación, la desigualdad y el desempleo. Todos ellos son factores que intervienen en

\footnotetext{
${ }^{1}$ Es importante destacar que el promedio de participación electoral en los 51 municipios que se maneja en este trabajo difiere del dato global -que es menor- reportado por la misma Comisión Electoral Estatal para los mismos años, debido a que la votación se cuenta institucionalmente con votos validados, no directamente de la lista nominal, ya que puede haber pérdidas en los conteos preliminares o simplemente casillas anuladas. Sin embargo, la tendencia a la baja se mantiene.

${ }^{2}$ La hipótesis del abandono es enunciada por Rosenstone (1982), quien sugiere que los problemas económicos provocan una decepción de los ciudadanos hacia el sistema político, haciéndolos menos propensos a la participación electoral.
} 
ciudadano al momento de decidir formar parte de un ejercicio democrático como son las elecciones para las alcaldías.

Se destaca el uso de datos georreferenciados de las variables analizadas usando fuentes secundarias, lo cual permite realizar el análisis espacial a fin de observar el posible efecto que pudiera tener la concentración o dispersión en el espacio del comportamiento de las variables en los municipios. ${ }^{3}$

De esta manera, si en un municipio específico predominan condiciones de elevados niveles de desigualdad, bajos niveles de escolaridad, altos niveles de violencia y elevados niveles de índices de marginación, es probable que esto incida en la participación de los ciudadanos en los comicios electorales. Y si a su vez, este municipio está rodeado de otros municipios con condiciones socioecómicas similares, entonces existirá adicionalmente un efecto espacial en la participación de los ciudadanos en las elecciones. Así, para los 51 municipios del estado de Nuevo León, se va a contrastar la relación que existe entre los componentes socioeconómicos, -analizando datos de desempleo, escolaridad, índice de Gini y la tasa de delitos violentos- con el porcentaje de participación electoral en el período histórico 2009-2018. ${ }^{4}$

Algunos de los resultados del análisis de cluster de la participación atestiguarán que existe dependencia espacial entre los diferentes municipios, siendo en las últimas elecciones una interacción municipal creciente en la zona metropolitana de Monterrey y municipios cercanos. Además, la violencia, el desempleo y el nivel de marginación alta al parecer tienen efectos en la participación electoral municipal.

Es importante indicar que existen estudios realizados para México como el de Vilalta (2008) y Hernández-Hernández (2015) en los cuales se concluye que los niveles de marginación sí están relacionados con las preferencias electorales.

La aportación de este estudio a la literatura es relevante por la poca o nula literatura con relación a los resultados electorales a nivel municipal en los cortes longitudinales por las metodologías propuestas, además de basarse para su análisis en información de fuentes secundarias. Los estudios más afines a este, si bien

\footnotetext{
${ }^{3}$ Es importante señalar que este análisis de la participación electoral se realiza a nivel municipal, contando además con información de desempleo, educación, desigualdad de ingresos, así como también a nivel desagregado. Así, este análisis implícitamente reporta una gran heterogeneidad de las regiones de Nuevo León, donde, por ejemplo, tenemos al área urbana y el área rural con sus grandes diferencias, pero además se aprovecha también esa relación de vecinos que algunos municipios tienen y que los caracterizan también. Es por esto que, por lo menos, aprovechando esta característica de dependencia espacial, se emplean herramientas de análisis espacial, siendo la parte de heterogeneidad que se aborda directamente en el modelo de regresión de error espacial para algunos años. ${ }^{4}$ Para la definición de las variables utilizadas, véase anexo.
} 
estudian el comportamiento electoral, no establecen una relación causal entre participación y características socieconómicas y se decantan mayormente por identificar la alineación partidaria en el Estado (Montero, 2019) o el modelo de competencia ideológica local, pero sólo para las elecciones federales de 2018 (León, 2019).

Las principales preguntas que se plantean son dos: ¿qué tipo de influencia tienen las variables socioeconómicas sobre la participación electoral?; ¿existe alguna influencia espacio-temporal de estas variables en la participación electoral? El análisis realizado nos permitirá explicar cómo afecta cada una de las variables socioecómicas a la participación electoral, controlada por la dependencia espacial.

Se establece como hipótesis principal que existe dependencia espacial de la participación electoral municipal; es decir, los municipios con altos niveles de participación electoral tienden a concentrarse territorialmente, mientras que esta circunstancia también se da a la inversa cuando disminuye la participación electoral.

De existir una relación entre la participación electoral y alguna de las variables socioeconómicas como educación, desempleo o desigualdad de ingresos, entonces existe una influencia espacial, por lo cual será necesario usar metodologías econométricas que permitan controlar la dependencia espacial para obtener parámetros objetivos de los efectos del entorno en la participación electoral.

Es por esto que en la segunda hipótesis establecemos tres relaciones entre la participación y las variables socioeconómicas analizadas. Estas son las siguientes:

1) La inseguridad afecta negativamente la participación electoral; esto es, a mayor violencia, menor participación electoral.

2) La cohesión social, medida como menor desigualdad económica a partir del Índice de Gini, favorece la participación electoral.

3) Un mayor nivel de marginación estimula la participación electoral.

Como ya se ha mencionado, en estas relaciones debemos de eliminar el presunto efecto de la dependencia espacial para obtener parámetros objetivos en los modelos que explican estos efectos.

El presente análisis se estructura de la siguiente manera: en la primera parte se establece el contexto regulatorio y la participación electoral en la entidad bajo análisis; en la segunda parte se presenta un marco teórico-empírico para explicar la participación electoral; en la tercera parte se muestra el enfoque espacial de los estudios empíricos relacionados con la participación; en la cuarta parte se establece la metodología y los datos; en la quinta parte se reporta el análisis exploratorio de los datos espaciales; en la sexta parte se establecen los resultados encontrados; en la séptima parte se realiza una discusión de los resultados; y, por último, en la octava parte se reportan las conclusiones. 


\section{CONTEXTO DE LAS REFORMAS Y PARTICIPACIÓN ELECTORAL EN NUEVO LEÓN}

Nuevo León ha vivido una experiencia que se podría denominar de transición política prolongada en donde los principales cambios han sido electorales, dentro de un proceso inacabado de consolidación democrática. Se advierte que en las diversas reformas electorales ocurridas entre 1976 y 2014 tanto a nivel local como a nivel federal han sido en función de las concesiones graduales que ha hecho el régimen político a las demandas de la oposición, tanto en el periodo príista como en el panista, tal y como ha ocurrido en el ámbito nacional (Medellín \& Murillo, 2014, pp. 19-30).

Las primeras reformas electorales -acaecidas en 1976, 1987 y 1991 - no quitaron la injerencia del gobierno en la organización de las elecciones. Solo se avanzó en el proceso de eficiencia electoral y de participación ciudadana del organismo electoral en las reformas siguientes -principalmente las de 1993 y 1996y que dieron paso al reemplazo interpartidario en 1997. Pero las reformas que siguieron en 1999, 2000 y 2002 continuaron siendo insuficientes y alimentaron de nuevo la demanda de continuar con el reformismo electoral. Por último, se señala que, si bien se avanzó en la reforma electoral en el año de 2007, sobre todo en temas de regulación de precampañas y de fiscalización de partidos políticos, la siguiente reforma fue un paso hacia adelante en criterios para la designación de autoridades electorales y jurisdiccionales (Alarcón \& García, 2016; Gilas, 2016; Marván, 2014). Esto es importante, porque esta coyuntura de reforma electoral ha generado, por lo menos, procesos equitativos de contienda, aunque los niveles de participación electorales se han mantenido estables.

En términos históricos, Medellín y Murillo (2014, p. 194) han mostrado que la participación electoral de los ciudadanos en el Estado en la década de los ochenta del siglo pasado no rebasó el 50\% de los electores inscritos. Sin embargo, entrados los años noventa, concretamente en 1994, se llegó hasta un 75\% coincidiendo con el asesinato del candidato presidencial Luis Donaldo Colosio. Después de ese etapa, la participación electoral se ha estabilizado en el período 20032012 entre en un 54 y un 60\% (Medellín \& Murillo, 2014, p. 196). Por eso, el análisis del proceso electoral puede estudiarse hoy más ampliamente a partir de estos resultados de participación electoral, cifras que son fiables en el período de tiempo que estamos señalando. 


\section{EXPLICANDO LA PARTICIPACIÓN ELECTORAL}

Uno de los puntos más importantes en la discusión sobre el desarrollo de la democracia es el tema de participación electoral. Definimos la participación electoral de acuerdo a Zineterer y Nohlen, como "el número de votos emitidos en una elección en relación con el número de personas con derecho a votar" (Zineterer \& Nohlen, 2006, p. 1002). Hay un consenso clásico sobre las corrientes más importantes para explicar la participación electoral como son la escuela de Columbia -enfocada en entender las condiciones sociales de los sujetos-, la escuela de Michigan centrada en variables explicativas de corte psicosocial- y la teoría de la elección racional (Montecinos, 2007; Muschick, 2004).

Otras explicaciones más recientes de la participación electoral están centradas estructuralmente bajo un orden clasificatorio distinto. Por ejemplo, Zineter y Nohlen las dividen en cuatro. En primer término, está el grado de igualdad social, ya que, bajo estos términos, una mayor desigualdad social tenderá en una más alta participación electoral de los estratos sociales bajos. En segundo lugar, lo que denominan concentración de cultura política en el Estado versus la sociedad. En tercer término, la centralidad del sistema representativo frente a otras arenas decisionales como el sistema de justicia o el administrativo. Por último, los autores remarcan que el grado de confianza en las instituciones políticas determina la participación electoral (Zineterer \& Nohlen, 2006, p. 1004).

Por otra parte, autores como Blais y Dobrzynska mantienen que hay factores que condicionan la participación electoral como el entorno socioeconómico -en consonancia con la escuela de Columbia- agregando además el entorno institucional y el sistema de partidos (1998, pp. 241-242). Bedoya et al. también coinciden en señalar variables institucionales para explicar el comportamiento electoral. Estas variables estarían definidas por el diseño del sistema electoral y de partidos, destacando elementos como la proporcionalidad del sistema, la obligatoriedad del voto, la simultaneidad de las elecciones, las leyes de registro, la fatiga electoral y el carácter bicameral o unicameral del parlamento (2019, p. 181). También desagregan otras variables explicativas como serían las dinámicas o las de violencia. Las primeras están referidas a la temporabilidad que se presentan en los comicios, el gasto en campañas o el uso de campañas negativas (2019, p. 185). Por otra parte, la última variable ha recibido también gran atención por la alta incidencia de la delincuencia y que influye negativamente con la instalación, el desarrollo y los niveles de votación en los procesos electorales, siendo paradigma de ello el caso mexicano (Hernández, 2010; Medellín \& Ontiveros, 2013; Valdez Zepeda \& Paniagua Vázquez, 2011). 
Ahora bien, si se pretende establecer correlaciones empíricas entre el desarrollo de la democracia y las variables de orden estructural, los especialistas refieren necesariamente la teoría de la modernización de Lipset, a pesar de las críticas que históricamente se han vertido sobre sus resultados (Redfield, 1965; Rostow, 1960). El mismo Lipset había sido precavido al advertir de que no se tomara de forma tan determinista una alta correlación entre las variables de la democracia -siendo un eje principal la participación electoral-y las características sociodemográficas de una sociedad en particular, ya que variables exógenas pueden derribar una correlación directa (2001, p. 116).

Autores que han criticado recientemente este modelo mencionan que para el caso mexicano existen relaciones inestables entre las bases socio-territoriales del desarrollo económico y el voto. Por lo tanto, no se puede aceptar de entrada un solo modelo de interpretación del comportamiento electoral y señalan la necesidad de medir el voto en diferentes contextos, niveles y escalas; más aún es evidente cuando se trata de comparar datos de comportamiento electoral en diferentes estados, no se observa un patrón único (Sonnleitner, 2007, p. 819; 2017, p. 419; Soto \& Cortez, 2014, p. 329). Tampoco en la literatura hay consenso sobre los efectos de la adversidad económica en la participación electoral, porque si bien se ha encontrado un efecto movilizador, también se ha verificado la hipótesis del abandono5; es decir, la adversidad económica provoca el descenso en la participación electoral (Bartle et al., 2017; Carreras \& Castañeda-Angarita, 2019; Lavezzolo, 2008).

Pese a estos diferendos en la literatura, queremos probar que, para el caso de Nuevo León, sí puede existir una alta correlación con las variables de orden económico. Tan solo haremos un ajuste al modelo, pues se pretende estimar el resultado de correlación entre variables con la participación electoral en el periodo citado, pero ligada a una variable determinante de la sociología política aplicada a una variante cuantitativa como es el análisis espacial.

Entendemos por sociología política el análisis explicativo de la formaciones político-sociales, siendo una derivación importante de su desarrollo la participación política y, por ende, el estudio del voto (Bobbio et al., 2015, pp. 15311532). En este sentido, el artículo se posiciona bajo el enfoque teórico de la escuela de Columbia, siendo uno de sus ejes de investigación explicar la participación electoral desde el entorno social (Lazarsfeld, P. et al.,1960).

\footnotetext{
${ }^{5}$ La hipótesis del abandono es enunciada por Rosenstone (1982) que sugiere que los problemas económicos provocan una decepción de los ciudadanos hacia el sistema político, haciéndola menos propensa a la participación electoral.
} 
En este sentido, la participación electoral también se ve favorecida en relación al nivel de desempleo, como lo encuentran en su estudio Burden y Wichowsky (2012), en el cual realizan un análisis a nivel estatal, por condados y a nivel individual en las elecciones del 2008 para Estados Unidos, en el cual hallan que los incrementos en los niveles de desocupación elevan la participación electoral.

Más específicamente, Cebula y Alexander (2017) realizan un análisis con datos panel para las cinco elecciones presidenciales de Estados Unidos entre 2000 a 2016, donde concluyen que al aumentar la participación laboral femenina se incrementa la participación de los votantes.

Pero también tiene su peso el argumento de la dependencia espacial, enfoque que conecta con la idea de que las personas que comparten un entorno tienden a adoptar una conducta política similar (Losada \& Casas, 2008, p. 157). Esto lo explicaremos en el siguiente apartado.

\section{EL ANÁLISIS DE AUTOCORRELACIÓN ESPACIAL}

El concepto de espacio ha sido ampliamente acogido por las ciencias sociales como un "[...] elemento fundamental en la comprensión de las sociedades humanas" (Mazurek, 2009, p. 10). En la literatura económica se ha estudiado ampliamente los efectos endógenos sociales en los cuales la propensión de un individuo a comportarse de una manera determinada varía con la prevalencia de la conducta de algún grupo de referencia con el que este tiene contacto. Dichos efectos se conocen como "normas sociales", "efectos vecindario", "imitación", "contagio" o "interacciones sociales" (Manski, 1993, p. 531).

Manski (1993) propone un análisis teórico para distinguir tres tipos de efectos a manera de hipótesis:

a) Endógenos, definidos como aquellos en que la propensión de un individuo a comportarse varía con la conducta del grupo.

b) Exógenos o aquellos en que la propensión de un individuo a comportarse de alguna manera varía con una característica exógena al grupo como, por ejemplo, el contexto social, económico.

c) Correlacionados, en donde los individuos en el mismo grupo tienden a comportarse similarmente porque ellos tienen características similares o enfrentan entornos institucionales similares.

En Elffers, el concepto de influencia -en un contexto de conducta criminal-entre los vecindarios conduce al uso de modelos de dependencia espacial, en los que la idea de adyacencia es clave, pues piensa que las características de un 
vecindario o región afectan a las características de los vecindarios o regiones adyacentes; esto es, áreas geográficas que comparten una frontera o bien que están a cierta distancia (Elffers, 2003).

Bajo este orden de ideas, consideramos que nos situamos en términos de la estructuración que de acuerdo a Giddens: "[...] se basa en la premisa de que este dualismo [entre sujeto y objeto social] se tiene que reconceptualizar como una dualidad: una dualidad de estructura" (2006, p. 22). Entiende por estructuración: "La articulación de relaciones sociales por un tiempo y un espacio, en virtud de la dualidad de estructura" (2006, p. 396). En ese sentido, las propiedades estructurales de los sistemas sociales existen en tanto las conductas sociales se reproducen en determinado contexto espacio-temporal. Es decir, la idea de estructuración permite el "descentramiento del sujeto" y las prácticas sociales, se consideran situadas tanto en la raíz de la constitución del sujeto como también del objeto social (2006, p. 23). Estamos de acuerdo con que los sistemas sociales organizan las relaciones y conductas sociales. Hasta aquí podemos pensar que estamos en la clásica teoría estructuralista parsoniana. Pero la variante introducida por Giddens es que son las mismas prácticas que pueden reconfigurar la continuidad de la estructura en la espacialidad-temporal y estas, a su vez, vuelven a incidir en la acción de las conductas sociales. Es un ciclo que no se rompe.

Si partimos de esta dimensión analítica, la reconfiguración tanto del objeto social - espacialidad territorial-y del sujeto - participación electoral y condiciones socieconómicas- en un período histórico - eje temporal-, podemos derivar la importancia de analizar los procesos electorales dentro del ámbito de la espacialidad. Si bien vinculamos variables explicativas del orden socioeconómico para la participación, puede ser también determinante la construcción de una determinada espacialidad que pueda influir en ese comportamiento electoral y viceversa.

En este sentido, Fiorino et al. (2016) realizaron para la Unión Europea un estudio sobre las elecciones parlamentarias entre 1999 a 2014. Observaron las disparidades regionales en la participación cuando hay diferencias en desempleo, edad del votante, PIB per cápita y transferencias financieras. En este estudio se encuentra que solo los ciudadanos de mayor edad de la población son más propensos -frente a los jóvenes- a participar en las elecciones. Estos investigadores usan, además de factores socioecómicos, institucionales y políticos, un modelo multinivel para su análisis espacial a nivel nacional y regional.

Existen pocos estudios para México, pero los que hay se refieren a las preferencias electorales del votante por los partidos políticos relacionando sus características socioeconómicas. Tal es el caso de Vilalta (2008) y Hernández- 
Hernández (2015). Así, por ejemplo, en el caso de Vilalta (2008), señala que para la Ciudad de México existen clusters espaciales de apoyo electoral y de marginación. Hernández-Hernández (2015) analiza las elecciones presidenciales de 2012 y señala que, por ejemplo, existen zonas rurales y ciudades medias con preferencias para un partido como el PRI con niveles de marginación bajos y medios.

Autores bajo este modelo interpretativo se han volcado en este tipo de análisis; es decir, en explicar cómo el espacio desempeña un papel estructurante, ya que este puede mediar en el cambio o la modulación de los procesos políticoelectorales (Lois, 2013, pp. 209, 210, 222-223; Pacheco, 2000, pp. 204-205; Vilalta, 2003; 2008).

También autores como Hägerstrand (1965), Cox (1969) y Huckfeldt y Sprague (1995) han establecido la importancia del contexto espacial que determina el efecto vecindario para procesar información y tomar decisiones políticas, siendo la más esencial la que se toma en el plano individual como es la hora de ir a votar. Sin embargo, hay un debate entre los especialistas sobre cuál es la escala más apropiada para medir los efectos, como han evidenciado los trabajos de Cutts y Fieldhouse (2009) y Weaver (2014). Por otra parte, O'Loughlin han relacionado la dependencia espacial en la difusión de los derechos civiles y valores democráticos (O'Loughlin, 2001, pp. 77-96).

Particularmente, este mismo autor ha definido el análisis espacial como el estudio de los datos que tiene una localización geográfica como, por ejemplo, recintos electorales, países, regiones, ciudades o atributos locales de los votantes como dirección de las calles, el lugar de trabajo o las redes personales ( $\mathrm{O}^{\prime}$ Loughlin, 2003, p. 43).

\section{Metodología y datos}

Se construyó una base de datos con información de los 51 municipios de Nuevo León con las principales variables socioeconómicas disponibles a nivel local, así como la participación electoral a nivel municipal para el periodo de 2009 a 2018.

Los datos usados de participación electoral municipal de 2009 a 2018 provienen de las Memorias de la Comisión Estatal Electoral de Nuevo León, mientras que las variables de escolaridad y desempleo son obtenidas del Censo de Población y Vivienda del INEGI de 2000 y 2010. 
TABLA 1 DEFINICIÓN DE VARIABLES

\begin{tabular}{|c|c|c|}
\hline Variable & Concepto & Definición \\
\hline PPE2009 & $\begin{array}{l}\text { Porcentaje de participación electoral } \\
2009\end{array}$ & $\begin{array}{l}\text { porcentaje de personas que votaron } \\
\text { respecto al total del padrón electo- } \\
\text { ral }\end{array}$ \\
\hline PPE2012 & $\begin{array}{l}\text { Porcentaje de participación electoral } \\
2012\end{array}$ & $\begin{array}{l}\text { porcentaje de personas que votaron } \\
\text { respecto al total del padrón electo- } \\
\text { ral }\end{array}$ \\
\hline PPE2015 & $\begin{array}{l}\text { Porcentaje de participación electoral } \\
2015\end{array}$ & $\begin{array}{l}\text { porcentaje de personas que votaron } \\
\text { respecto al total del padrón electo- } \\
\text { ral }\end{array}$ \\
\hline PPE2018 & $\begin{array}{l}\text { Porcentaje de participación electoral } \\
2018\end{array}$ & $\begin{array}{l}\text { porcentaje de personas que votaron } \\
\text { respecto al total del padrón electo- } \\
\text { ral }\end{array}$ \\
\hline escol2010 & Grado promedio de escolaridad 2010 & años promedio de escolaridad \\
\hline escol2015 & Grado promedio de escolaridad 2015 & años promedio de escolaridad \\
\hline escol2000 & Grado promedio de escolaridad 2000 & años promedio de escolaridad \\
\hline desemp2010 & Tasa de desempleo (\%)_2010 & $\begin{array}{l}\text { porcentaje de personas desocupa- } \\
\text { das respecto a la población econó- } \\
\text { micamente activa }\end{array}$ \\
\hline desemp2000 & Tasa de desempleo (\%)_2000 & $\begin{array}{l}\text { porcentaje de personas desocupa- } \\
\text { das respecto a la población econó- } \\
\text { micamente activa }\end{array}$ \\
\hline tviol2011 & $\begin{array}{l}\text { Tasa de delitos violentos por cada mil } \\
\text { personas_2011 }\end{array}$ & $\begin{array}{l}\text { [(total de incidencia delictiva por } \\
\text { delitos de homicidio, robo con vio- } \\
\text { lencia, violación, violencia fami- } \\
\text { liar)/población total)] }{ }^{*} 1000\end{array}$ \\
\hline tviol2015 & $\begin{array}{l}\text { Tasa de delitos violentos por cada mil } \\
\text { personas_2015 }\end{array}$ & $\begin{array}{l}\text { [(total de incidencia delictiva por } \\
\text { delitos de homicidio, robo con vio- } \\
\text { lencia, violación, violencia fami- } \\
\text { liar)/(población total)]*1000 }\end{array}$ \\
\hline gini2010 & Coeficiente de Gini 2010 & $\begin{array}{l}\text { Rango de } 0 \text { a 1, entre más cercano } \\
\text { a } 1 \text { es mayor la desigualdad }\end{array}$ \\
\hline gini2015 & Coeficiente de Gini 2015 & $\begin{array}{l}\text { Rango de } 0 \text { a } 1 \text {, entre más cercano } \\
\text { a } 1 \text { es mayor la desigualdad }\end{array}$ \\
\hline d_maralta00 & $\begin{array}{l}\text { Dummy de grado de marginación alta } \\
2000\end{array}$ & $\begin{array}{l}\text { es } 1 \text { si tiene marginación alta, } 0 \text { si } \\
\text { no }\end{array}$ \\
\hline d_marbaj00 & $\begin{array}{l}\text { Dummy de grado de marginación baja } \\
2000\end{array}$ & $\begin{array}{l}\text { es } 1 \text { si tiene marginación baja, } 0 \text { si } \\
\text { no }\end{array}$ \\
\hline d_marmed00 & $\begin{array}{l}\text { Dummy de grado de marginación me- } \\
\text { dia } 2000\end{array}$ & $\begin{array}{l}\text { es } 1 \text { si tiene marginación media, } 0 \\
\text { si no }\end{array}$ \\
\hline d_maralta10 & $\begin{array}{l}\text { Dummy de grado de marginación alta } \\
2010\end{array}$ & $\begin{array}{l}\text { es } 1 \text { si tiene marginación alta, } 0 \text { si } \\
\text { no }\end{array}$ \\
\hline
\end{tabular}




\begin{tabular}{|l|l|l|}
\hline d_marbaj10 & $\begin{array}{l}\text { Dummy de grado de marginación baja } \\
2010\end{array}$ & $\begin{array}{l}\text { es 1 si tiene marginación baja, 0 si } \\
\text { no }\end{array}$ \\
\hline d_marmed10 & $\begin{array}{l}\text { Dummy de grado de marginación me- } \\
\text { dia 2010 }\end{array}$ & $\begin{array}{l}\text { es 1 si tiene marginación media, 0 } \\
\text { si no }\end{array}$ \\
\hline d_maralta15 & $\begin{array}{l}\text { Dummy de grado de marginación alta } \\
2015\end{array}$ & $\begin{array}{l}\text { es 1 si tiene marginación alta, 0 si } \\
\text { no }\end{array}$ \\
\hline d_marbaj15 & $\begin{array}{l}\text { Dummy de grado de marginación baja } \\
2015\end{array}$ & $\begin{array}{l}\text { es 1 si tiene marginación baja, 0 si } \\
\text { no }\end{array}$ \\
\hline d_marmed15 & $\begin{array}{l}\text { Dummy de grado de marginación me- } \\
\text { dia 2015 }\end{array}$ & $\begin{array}{l}\text { es 1 si tiene marginación media, 0 } \\
\text { si no }\end{array}$ \\
\hline
\end{tabular}

Fuente: Elaboración propia con datos de la Comisión Estatal Electoral de Nuevo León (2010, 2013, 2016, 2019); Instituto Nacional de Geografía y Estadística (2000, 2010); Secretariado Ejecutivo del Sistema Nacional de Seguridad Pública (2019); Consejo Nacional de Evaluación de la Política de Desarrollo Social (2015a, 2015b).

La información de delitos a nivel municipal es obtenida de las estadísticas del Secretariado Ejecutivo del Sistema Nacional de Seguridad Pública (SESNSP) para los años de 2011 y 2015. Mientras que el Índice de Marginación a Nivel Municipal es tomado del Consejo Nacional de Evaluación de la Política de Desarrollo Social (CONEVAL) para los años 2000, 2010 y 2015, al igual que el Índice de Gini. En la siguiente tabla se definen las variables utilizadas en el presente estudio.

Ahora bien, el concepto de autocorrelación es definido como la correlación entre los componentes de una serie de observaciones ordenadas en el tiempo -como en los datos de series de tiempo- o en el espacio -como en los datos de corte transversal- (Gujarati, 2004, p. 441). La autocorrelación espacial o la coincidencia de valores similares con similaridad local puede ser formalmente expresada como la condición de

$$
\operatorname{Cov}\left[y_{i}, y_{j}\right]=E\left[y_{i} y_{j}\right]-E\left[y_{i}\right]^{*} E\left[y_{j}\right] \neq 0 \text {, para } i \neq j
$$

Donde la ecuación (1) es la covarianza; i,j se refieren a las observaciones individuales (ubicaciones), yi(j) es el valor de una variable aleatoria de interés en esa ubicación. Esta covarianza se convierte en un significado relevante desde la perspectiva espacial cuando la configuración particular de no-cero de las observaciones $\mathrm{i}, \mathrm{j}$ tiene una interpretación en términos de la estructura espacial, la interacción espacial o el arreglo espacial de las observaciones (Anselin, 1999, p. 4).

En el conjunto de las observaciones recolectadas en los datos de corte transversal, hay suficiente información para estimar una matriz de $\mathrm{NxN}$, donde $\mathrm{N}$ es el total de regiones o municipios, la cual se conoce como matriz de pesos espaciales. Esta matriz especifica la relación de vecindario entre los municipios, la cual es obtenida especificando un 1 para cada ubicación i -en la fila de la matriz- 
del vecino en las columnas correspondientes, y si no hay vecindad se le asigna $0^{6}$. En este estudio se utilizó la matriz de pesos espaciales de distancia inversa.

Respecto a las técnicas de autocorrelación espacial, también se disponen desde diferentes perspectivas, dependiendo de que el caso analizado sea univariante o multivariable.

En el caso univariable, la prueba más utilizada para la autocorrelación espacial es el indicador de I de Moran (Anselin, 1999), que es un estadístico de asociación global de autocorrelación espacial y es la aproximación más tradicional al efecto de dependencia espacial, permitiendo contrastar la presencia o ausencia de un esquema de dependencia espacial a nivel de una variable; es decir, contrasta si se cumple la hipótesis de que una variable se encuentra distribuida con un patrón aleatorio en el espacio o si, por el contrario, existe una asociación significativa de valores similares o diferentes entre regiones vecinas (Moreno \& Vaya, 2002).

La ecuación 2 específica este indicador.

$$
I=\frac{N}{S_{0}} \frac{\sum w_{i j}\left(y_{i}-\bar{y}\right)\left(y_{j}-\bar{y}\right)}{\sum_{i=1}^{N}\left(y_{i}-\bar{y}\right)^{2}}
$$

Donde

wij: es elemento de la matriz de pesos espaciales al par $(\mathrm{i}, \mathrm{j})$

$\mathrm{S} 0=$ es la suma total de los pesos espaciales

$\bar{y}=$ valor medio o esperado de la variable $\mathrm{y}$

$\mathrm{N}=$ número de observaciones

Anselin, citado en Chasco (2003), propone un conjunto de indicadores locales de asociación espacial LISA -Local Indicators of Spatial Associationcapaces de detectar la contribución de cada región a un indicador de dependencia espacial global como, por ejemplo, el I de Moran. Este tipo de indicadores permiten identificar los agrupamientos espaciales locales significativos en torno a un punto concreto en el espacio - la formación de clusters- y la presencia de valores atípicos; es decir, la inestabilidad espacial.

Por otro lado, para el efecto de dependencia espacial en el caso de análisis multivariable se utilizan los modelos espaciales que son aquellos modelos de regresión lineal que consideran explícitamente la existencia del efecto espacial de dependencia o autocorrelación, ya que la presencia de la misma invalida la inferencia estadística derivada de la estimación por el tradicional método de Mínimo

\footnotetext{
${ }^{6}$ Por convención, a una misma región se le asigna también 0. Para ver más del tipo de interacción que se puede especificar en la matriz de pesos espaciales, véase Anselin (1999).
} 
Cuadrados Ordinarios - $\mathrm{MCO}$-. La ventaja es que este tipo de modelos permite detectar fenómenos como spillover -desbordamientos- en una determinada unidad espacial (Chasco, 2003).

El efecto de dependencia espacial puede estar presente en un modelo en forma de autocorrelación espacial en alguna variable del mismo -endógena y/o exógena-, se le conoce como Modelo Autorregresivo de Regresión Espacial de orden $1-\operatorname{SAR}(1-$ :

Donde:

$$
\begin{gathered}
y=\rho W y+u(3) \\
u \approx N\left(0, \sigma^{2} I\right)
\end{gathered}
$$

$\mathrm{y}$ : es un vector $(\mathrm{N} x \mathrm{l})$ de observaciones de la variable endógena

$\mathrm{W}$ : es la matriz de pesos espaciales de la variable endógena -normalizada-, siendo "Wy" el retardo espacial de la variable endógena

$\rho$ : coeficiente autorregresivo espacial - escalar-, que recoge la intensidad de las interdependencias entre las observaciones muestrales

u: es la perturbación aleatoria de ruido blanco.

Pero también pueden existir esquemas de dependencia espacial en el término de la perturbación aleatoria. A este tipo de modelos se le conoce como Modelo de Error Espacial -MEE-. La existencia de ciertos factores o variables no explícitamente considerados en el modelo trasladan hacia los términos del error la configuración de agrupación de valores -autocorrelación- presente en la variable endógena, que también suele especificarse como un proceso autorregresivo de orden 1 o AR(1). La ecuación 4 especifica este tipo de modelo (Chasco, 2003):

Donde:

$$
\begin{gathered}
y=X \beta+u(4) \\
u=\lambda W u+\epsilon \\
\epsilon \approx N\left(0, \sigma^{2} I\right)
\end{gathered}
$$

$\mathrm{u}$ : es la perturbación aleatoria distribuida según un proceso AR(1)

$\lambda$ : es el parámetro autorregresivo (escalar) asociado al retardo espacial $\mathrm{Wu}$

$\epsilon$ : es el vector de perturbaciones aleatorias que es de ruido blanco.

Es importante señalar que, dependiendo del comportamiento de los datos, se aplicó el modelo de SAR1, donde la interacción espacial entre las unidades observadas está determinada por la variable endógena, en este caso de la participación electoral municipal o bien donde la dependencia espacial proviene del término de error, por lo cual se aplica en su caso $\mathrm{MEE}^{7}$.

\footnotetext{
${ }^{7}$ Se utilizó el software GEODA (Geographic Data Analysis) para el análisis de las regresiones, por lo cual el tipo de modelo está limitado al uso de los modelos SAR1 o MEE.
} 


\section{ANÁLISIS EXPLORATORIO ESPACIAL (AEE)}

Se presenta un análisis descriptivo de la distribución en el espacio de las variables que son objeto de análisis, con el fin de identificar localizaciones atípicas-outliers espaciales-, descubrir esquemas de asociación espacial -cluster espacial- y, con esto, sugerir el tipo de modelo que explique ese comportamiento. El centro de todos estos conceptos lo ocupa la autocorrelación espacial. Para esto, se estimarán dos indicadores de dependencia espacial univariable: el I de Moran y el Î́ndice de Autocorrelación Espacial Local o LISA, por sus siglas en inglés.

En lo que respecta al análisis descriptivo, se observa que la media de participación electoral en 2009 fue de $65.03 \%$, mientras que en 2018 esta participación fue de $63.25 \%$. El promedio de escolaridad en los 51 municipios en 2000 fue de 6.9 años, mientras que en 2015 este indicador educativo fue de 8.4 años de escolaridad.

El desempleo se incrementó entre 2000 y 2010 en promedio para todos los municipios del Estado, mientras que la tasa de delitos violentos en 2001 fue de 4.4 por cada mil habitantes, en tanto que en 2015 fue de 2.97 delitos por cada mil personas. El 12\% de los municipios presentó un grado de marginación alta en 2000, mientras que este indicador para 2015 fue de $8 \%$. Por otro lado, el índice de Gini -indicador de desigualdad-pasó de 0.38 en 2010 a 0.39 en 2015. Este indicador oscila en un rango de 0 a 1 . Cuanto más cercano a 1 indica un mayor grado de desigualdad y, por lo tanto, menos cohesión social.

De acuerdo a la tabla 1, se reporta que casi todas las variables involucradas en este estudio presentan autocorrelación espacial positiva y es significativa al nivel del 5\%, sobre todo las variables relacionadas a la participación electoral para cada año analizado.

Con los mapas del indicador de autocorrelación espacial local municipal se puede detectar la presencia de la dependencia espacial en la participación electoral a nivel municipal. Desde 2009 a 2018 se observa como el patrón de dependencia espacial ha sido creciente en la formación de clusters o agrupaciones de municipios respecto a la participación electoral. Por ejemplo, se aprecia como los municipios del área metropolitana de Monterrey y los cercanos a ellos, tienen una dependencia espacial positiva cada vez más creciente en los cuatro años.

Esto parece indicar que los municipios del área metropolitana de Monterrey y cercanos a ellos que tienen bajos niveles de participación electoral, tienen municipios vecinos que también tienen bajos niveles de participación electoral. Los municipios del norte y sur del estado también muestran una dependencia 
espacial positiva; esto es, los municipios con altos niveles de participación electoral tienen vecinos que también tienen alta participación electoral ${ }^{8}$.

TABLA 2 ANÁLISIS DESCRIPTIVO DE VARIABLES

\begin{tabular}{|c|c|c|c|c|c|c|}
\hline Variable & Obs & Media & Desv. Est. & Min & $\operatorname{Max}$ & I Moran \\
\hline PPE_2009 & 51 & 65.03 & 10.01 & 46.88 & 86.95 & $* 0.241$ \\
\hline PPE_2012 & 51 & 67.43 & 9.58 & 50.91 & 87.99 & $* 0.331$ \\
\hline PPE_2015 & 51 & 67.66 & 10.49 & 50.51 & 89.41 & $* 0.386$ \\
\hline PPE_2018 & 51 & 63.25 & 13.78 & 32.86 & 87.29 & $* 0.488$ \\
\hline escol2010 & 51 & 7.87 & 1.41 & 5.30 & 12.09 & $* 0.582$ \\
\hline escol2015 & 51 & 8.36 & 1.41 & 5.73 & 12.80 & $* 0.588$ \\
\hline escol2000 & 51 & 6.88 & 1.34 & 4 & 11 & $* 0.502$ \\
\hline desemp 2010 & 51 & 3.94 & 1.58 & 0.670 & 7.739 & $* 0.220$ \\
\hline desemp2000 & 51 & 1.49 & 0.68 & 0.543 & 4.382 & 0.039 \\
\hline d_maralt00 & 51 & 0.12 & 0.33 & 0 & 1 & $* 0.678$ \\
\hline d_marmed00 & 51 & 0.04 & 0.20 & 0 & 1 & -0.016 \\
\hline d_marbaj00 & 51 & 0.84 & 0.37 & 0 & 1 & $* 0.654$ \\
\hline d_maralt10 & 51 & 0.02 & 0.14 & 0 & 1 & -0.021 \\
\hline d_marmed 10 & 51 & 0.12 & 0.33 & 0 & 1 & -0.076 \\
\hline d_marbaj10 & 51 & 0.86 & 0.35 & 0 & 1 & -0.023 \\
\hline d_maralt15 & 51 & 0.08 & 0.27 & 0 & 1 & $* 0.313$ \\
\hline d_marmed 15 & 51 & 0.06 & 0.24 & 0 & 1 & -0.045 \\
\hline d_marbaj15 & 51 & 0.86 & 0.35 & 0 & 1 & $* 0.767$ \\
\hline tviol2011 & 51 & 4.43 & 3.09 & 0.281 & 15.728 & $* 0.195$ \\
\hline tviol2015 & 51 & 2.97 & 1.86 & 0.493 & 10.699 & 0.044 \\
\hline gini10 & 51 & 0.38 & 0.04 & 0.289 & 0.501 & $* 0.512$ \\
\hline gini15 & 51 & 0.39 & 0.05 & 0.303 & 0.494 & $* 0.514$ \\
\hline
\end{tabular}

*Significativo al 5\%. Fuente: Elaboración propia con datos de la Comisión Estatal Electoral de Nuevo León (2010, 2013, 2016, 2019); Instituto Nacional de Geografía y Estadística (2000, 2010); Consejo Nacional de Evaluación de la Política de Desarrollo Social. (2015a, 2015b).

\footnotetext{
${ }^{8}$ En el anexo 1 se presenta un mapa municipal del Estado de Nuevo León.
} 
FiguRA 1. INDICADOR LOCAL ESPACIAL DE LA PARTICIPACIÓN EleCtORAL EN EL ESTADO DE NUEVO LEÓN

2009 (I DE MORAN=0.241)

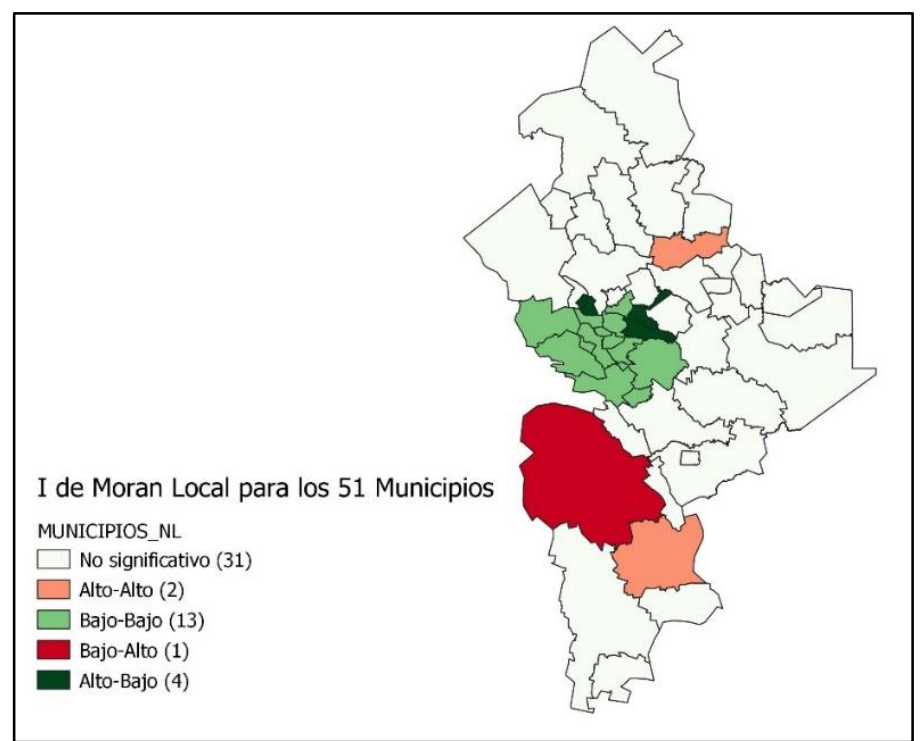

2012 (I DE MORAN=0.331)

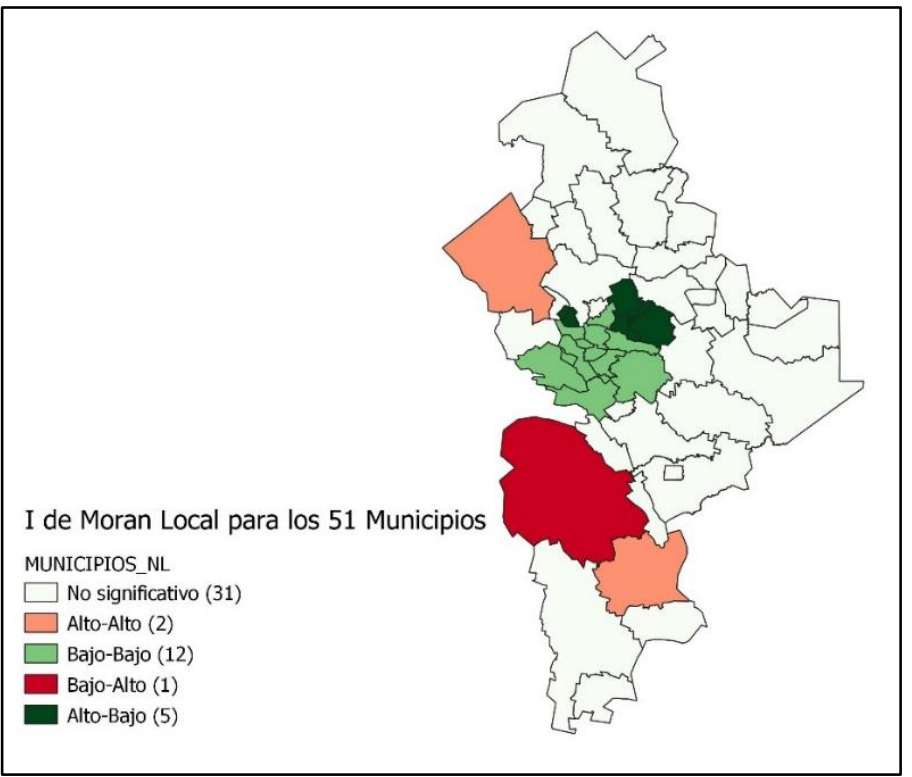


$2015($ I DE MORAN=0.386)

I de Moran Local para los 51 Municipios

MUNICIPIOS_NL

No significativo (27)

Alto-Alto (3)

Bajo-Bajo (18)

Bajo-Alto (0)

Alto-Bajo (3)

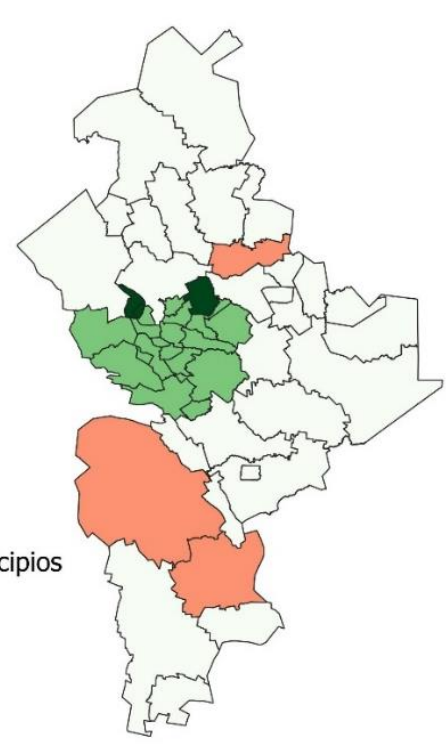

2018 (I DE MORAN=0.488)

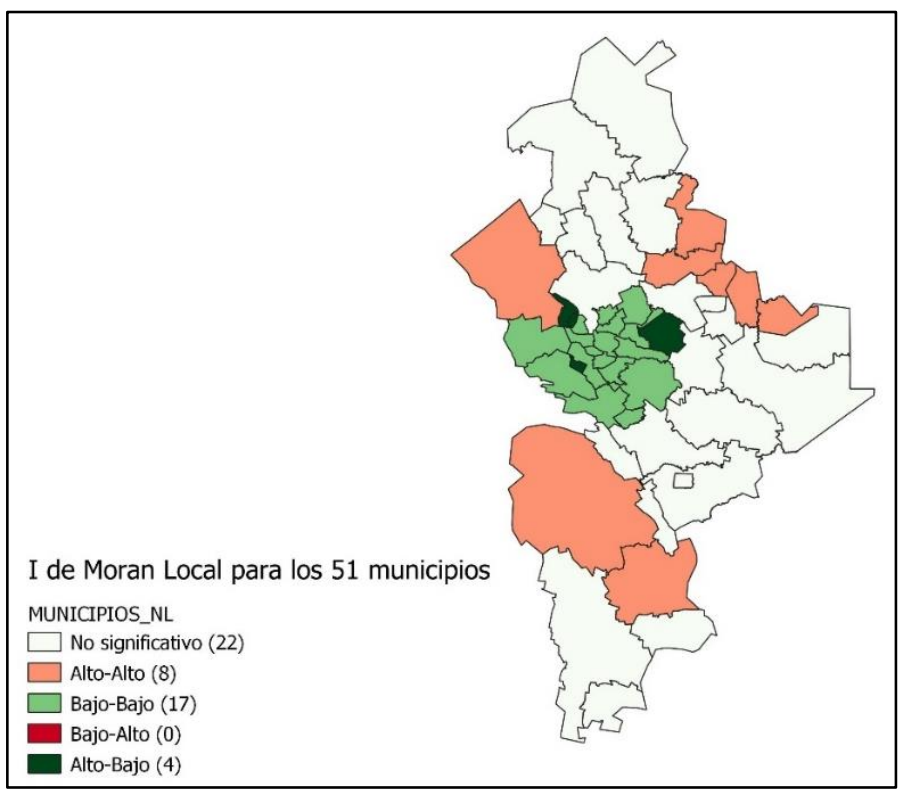

Fuente: Elaboración propia. La matriz de pesos espaciales usada es distancia inversa. Todos los resultados reportaron significancia entre el $1 \%$ y $10 \%$ con un nivel de 999 permutaciones. Entre paréntesis se reporta el número de municipios. 
Lo destacable de este análisis de cluster dado por el indicador LISA, es que se observan, a lo largo del procesos electorales en la región, significativas concentraciones de participación electoral alta en los municipios del sur y del norte -área roja-, ya que las participaciones electorales han sido cada vez más crecientes entre municipios; es decir, cada vez son más los municipios que tienen una participación electoral elevada y cuentan con vecinos en que también su participación es elevada.

\section{RESULTADOS DEL MODELO ELECTORAL}

Se estimaron cuatro tipos de modelo debido a que incluir todas las variables en un solo modelo tenía problemas de multicolinealidad; es decir, que entre las variables explicativas existe relación, por lo cual los parámetros corren el riesgo de estar sesgados.

En general las estimaciones se hicieron tanto al nivel de las variables, pero, para facilitar la interpretación de los resultados, se estimó la variable dependiente en logaritmo natural haciendo una regresión semilog.

El modelo A es un modelo donde sólo se relaciona la participación electoral con las variables de desempleo, escolaridad y delitos violentos. En el modelo $\mathrm{B}$, además de las variables anteriores, se incluye el nivel de desigualdad de la población medida con el índice Gini. En el modelo C, se incluyen las variables del modelo A, agregando solamente el Índice de Rezago Social con variables dicotómicas, clasificando este índice en bajo, medio y alto. Por último, el modelo $\mathrm{D}$, es el modelo de regresión espacial, el cual corrige las estimaciones del modelo que mejor explica los datos -se tomó la mayor $\mathrm{R}^{2}$ ajustada, además de los indicadores de Aikaike y Schwarz-, que en este caso fue el modelo C semilog.

Para todos los modelos, las variables independientes a nivel municipal fueron la tasa de desempleo abierto, el nivel de escolaridad promedio medido en años, la tasa de incidencia de delitos violentos - delitos como homicidio, robo con violencia, violación, violencia familiar-por cada mil habitantes, el coeficiente de Gini y el Índice de Marginación clasificado en bajo, medio y alto.

\section{Elecciones 2009}

En la participación electoral en 2009 para los 51 municipios, solamente dos variables socioeconómicas resultaron significativas sin considerar la dependencia espacial. Estas fueron el nivel de educación y el grado de marginación alta. De acuerdo a los resultados de los modelos A y B, a mayor nivel de educación promedio, la participación electoral disminuye para este periodo, mientras que la participación electoral aumenta si los municipios tienen un grado mayor 
de marginación alta que cuando tienen un grado de marginación baja. Es de destacar como en todos los modelos de regresión, el indicador de I Moran resultó altamente significativo, lo que indica la presencia de dependencia espacial en los datos de regresión y que, de no tomarla en cuenta, los coeficientes resultan estar sesgados. Una vez considerada la dependencia espacial, el modelo que mejor la explica es el de Error Espacial -modelo C-, que solo reporta el grado de marginación baja como el que más influye positivamente en el comportamiento electoral; esto es, si en un municipio el grado de marginación alta aumenta en un 1\%, la participación electoral también aumenta en promedio en 0.19 puntos, comparado un municipio que tiene baja marginación; además, el coeficiente lambda que indica el control por el efecto espacial resulta significativo en el modelo.

Es importante destacar el alto grado de explicación del modelo espacial en relación a los modelos tradicionales de regresión.

TABla 3. REsultados 2009

\begin{tabular}{|c|c|c|c|c|c|}
\hline \multicolumn{6}{|c|}{ Variable dependiente: Participación electoral (\%) 2009} \\
\hline \multirow{2}{*}{$\begin{array}{l}\text { Variable } \\
\text { explicativa }\end{array}$} & \multicolumn{2}{|c|}{ Modelo A } & \multicolumn{2}{|c|}{ Modelo B } & \multirow{2}{*}{$\frac{\text { Modelo C }}{\text { Modelo Error Espacial }}$} \\
\hline & Nivel & Semilog & Nivel & Semilog & \\
\hline desemp2000 & $\begin{array}{r}1.785 \\
(0.3527)\end{array}$ & $\begin{array}{r}0.026 \\
(0.3827)\end{array}$ & $\begin{array}{r}2.275 \\
(0.2406)\end{array}$ & $\begin{array}{r}0.033 \\
(0.2723)\end{array}$ & $\begin{array}{r}1.127 \\
(0.4567)\end{array}$ \\
\hline escol2000 & $\begin{array}{r}*-3.420 \\
(0.0009) \\
\end{array}$ & $\begin{array}{r}*-\mathbf{0 . 0 5 4} \\
(0.0007)\end{array}$ & $\begin{array}{r}* * *-2.099 \\
(0.0828) \\
\end{array}$ & $\begin{array}{r}* * * \mathbf{0 . 0 3 6} \\
(0.0566) \\
\end{array}$ & $\begin{array}{r}\mathbf{- 0 . 1 5 2} \\
(0.9057) \\
\end{array}$ \\
\hline d_maralta2000 & & & $\begin{array}{r}* * * \mathbf{8 . 0 4 9} \\
(0.0908) \\
\end{array}$ & $\begin{array}{r}0.108 \\
(0.1419) \\
\end{array}$ & $\begin{array}{r}* * \mathbf{1 9 . 9 8 3} \\
(0.0011) \\
\end{array}$ \\
\hline d_marmed2000 & & & $\begin{array}{r}7.398 \\
(0.2666) \\
\end{array}$ & $\begin{array}{r}0.109 \\
(0.2895) \\
\end{array}$ & $\begin{array}{r}6.842 \\
(0.2449) \\
\end{array}$ \\
\hline Constante & $\begin{array}{r}85.899 \\
(0) \\
\end{array}$ & $\begin{array}{r}4.495 \\
(0) \\
\end{array}$ & $\begin{array}{r}74.842 \\
(0) \\
\end{array}$ & $\begin{array}{r}4.344 \\
(0) \\
\end{array}$ & $\begin{array}{r}63.399 \\
(0) \\
\end{array}$ \\
\hline Lambda & & & & & $\begin{array}{r}* \mathbf{0 . 6 1 6} \\
(0) \\
\end{array}$ \\
\hline $\mathrm{N}$ & 51 & 51 & 51 & 51 & 51 \\
\hline $\mathcal{R}^{2}$ ajustada & 0.222 & 0.227 & 0.247 & 0.241 & 0.449 \\
\hline Aikake criterion & 369.79 & -56.26 & 369.90 & -55.35 & 363.45 \\
\hline Schwarz criterion & 375.59 & -50.46 & 379.56 & -45.69 & 373.10 \\
\hline I de Moran & $\begin{array}{l}* * 1.92 \\
(0.0552) \\
\end{array}$ & $\begin{array}{l}* * * 1.93 \\
(0.0530) \\
\end{array}$ & $\begin{array}{r}* * \mathbf{2 . 3 8} \\
(0.0174) \\
\end{array}$ & $\begin{array}{r}* * \mathbf{2 . 3 9} \\
(0.0170) \\
\end{array}$ & \\
\hline
\end{tabular}

Se reporta para cada modelo los resultados a nivel de las unidades de la variables (NIVEL) y los resultados en términos de unidades logarítmicas en las variables independientes (Semilog) ${ }^{9}$.

Nota: entre paréntesis se reporta el $p$-value.

* Significativo al 1\%, **Significativo al 5\% y ***Significativo al $10 \%$

Fuente: Estimaciones propia

\footnotetext{
${ }^{9}$ En el modelo semilog, los coeficientes obtenidos son semielasticidades, por lo cual, para estimar el cambio absoluto de la variable dependiente ante un cambio porcentual de un $1 \%$ de la variable independiente, se deberá de multiplicar por 0.01 el coeficiente (Guajarati, 2004).
} 


\section{Elecciones 2012}

Se tomó como variable dependiente el porcentaje de participación electoral (PPE) de las elecciones del 2012 a nivel municipal, siendo las variables independientes la tasa porcentual de desempleo del 2000, el nivel promedio de escolaridad del 2000 -en años-, la tasa de incidencia de delitos violentos por cada mil personas en 2011, el Índice Gini para 2010 y el índice de Rezago Social clasificado en alto, medio y bajo.

TABla 4 Resultados 2012

\begin{tabular}{|c|c|c|c|c|c|c|c|}
\hline \multicolumn{8}{|c|}{ Variable dependiente: Participación electoral (\%) 2012} \\
\hline \multirow{2}{*}{$\begin{array}{l}\text { Variable } \\
\text { explicativa }\end{array}$} & \multicolumn{2}{|c|}{ Modelo A } & \multicolumn{2}{|c|}{ Modelo B } & \multicolumn{2}{|c|}{ Modelo C } & \multirow{2}{*}{$\begin{array}{c}\text { Modelo D } \\
\text { Modelo } \\
\text { Error } \\
\text { Espacial }\end{array}$} \\
\hline & Nivel & Semilog & Nivel & Semilog & Nivel & Semilog & \\
\hline desemp2000 & $\begin{array}{r}* * * 3.26 \\
6 \\
(0.0885) \\
\end{array}$ & $\begin{array}{r}0.045 \\
(0.1139)\end{array}$ & $\begin{array}{r}* * \mathbf{3 . 7 5 6} \\
(0.0282)\end{array}$ & $\begin{array}{r}* * * \mathbf{0 . 0 5 2} \\
(0.0406)\end{array}$ & $\begin{array}{r}* * \mathbf{3 . 6 7 0} \\
(0.0287)\end{array}$ & $\begin{array}{r}* * \mathbf{0 . 0 5 1 6} \\
(0.0416)\end{array}$ & $\begin{array}{r}* * \mathbf{0 . 0 3 5} \\
(0.0882)\end{array}$ \\
\hline escol2000 & $\begin{array}{l}* *-2.411 \\
(0.0161)\end{array}$ & $\begin{array}{r}* * \mathbf{0 . 0 3 5} \\
(0.0193)\end{array}$ & & & & & \\
\hline tviol2011 & $\begin{array}{r}-0.469 \\
(0.2522)\end{array}$ & $\begin{array}{r}-0.006 \\
(0.3489)\end{array}$ & $\begin{array}{r}-0.425 \\
(0.2534)\end{array}$ & $\begin{array}{r}-0.005 \\
(0.3692)\end{array}$ & $\begin{array}{r}-0.068 \\
(0.8551)\end{array}$ & $\begin{array}{r}0.000 \\
(0.9928)\end{array}$ & $\begin{array}{r}0.003 \\
(0.5296)\end{array}$ \\
\hline Gini2010 & & & $\begin{array}{r}* \mathbf{1 1 3 . 7 8 4} \\
(0.0002)\end{array}$ & $\begin{array}{r}* 1.70221 \\
(0.0002)\end{array}$ & & & \\
\hline d_maralta2000 & & & & & $\begin{array}{r}* \mathbf{1 6 . 1 9 1} \\
(0.00004)\end{array}$ & $\begin{array}{r}* \mathbf{0 . 2 2 8} \\
(0.0001)\end{array}$ & $\begin{array}{r}* \mathbf{0 . 2 8 1} \\
(0.00002)\end{array}$ \\
\hline d_marmed2000 & & & & & $\begin{array}{r}9.191 \\
(0.1081)\end{array}$ & $\begin{array}{r}0.139 \\
(0.1091)\end{array}$ & $\begin{array}{r}0.096 \\
(0.1964)\end{array}$ \\
\hline Constante & $\begin{array}{r}81.224 \\
(0)\end{array}$ & $\begin{array}{r}4.399 \\
(0)\end{array}$ & $\begin{array}{l}20.307 \\
(0.072)\end{array}$ & $\begin{array}{r}3.497 \\
(0)\end{array}$ & $\begin{array}{r}59.985 \\
(0)\end{array}$ & $\begin{array}{r}4.092 \\
(0)\end{array}$ & $\begin{array}{r}4.108 \\
(0)\end{array}$ \\
\hline Lambda & & & & & & & $\begin{array}{r}\boldsymbol{* 0 . 5 0 4} \\
(0.0006)\end{array}$ \\
\hline $\mathrm{N}$ & 51 & 51 & 51 & 51 & 51 & 51 & 51 \\
\hline $\mathcal{R}^{2}$ ajustada & 0.19 & 0.17 & 0.32 & 0.31 & 0.37 & 0.33 & 0.50 \\
\hline Aikake criterion & 368.04 & -61.08 & 359.01 & -70.79 & 356.16 & -71.29 & -78.12 \\
\hline $\begin{array}{l}\text { Schwarz } \\
\text { criterion }\end{array}$ & 375.80 & -53.35 & 366.74 & -63.06 & 365.82 & -61.63 & -68.46 \\
\hline I de Moran & $\begin{array}{r}* \mathbf{2 . 6 2 3 3} \\
(0.009)\end{array}$ & $\begin{array}{r}* \mathbf{2 . 5 6 4 7} \\
(0.010)\end{array}$ & $\begin{array}{r}* * \mathbf{2 . 2 8 8 7} \\
(0.022)\end{array}$ & $\begin{array}{r}* * \mathbf{2 . 3 8 0 2} \\
(0.0173)\end{array}$ & $\begin{array}{r}* \mathbf{2 . 9 8 8 9} \\
(0.0028)\end{array}$ & $\begin{array}{r}* \mathbf{2 . 9 2 8 8} \\
(0.0034)\end{array}$ & \\
\hline
\end{tabular}

Se reporta para cada modelo los resultados a nivel de las unidades de la variables (NIVEL) y los resultados en términos de unidades logarítmicas en las variables independientes (Semilog).

Nota: entre paréntesis se reporta el p value

* Significativo al 1\%, **Significativo al $5 \%$ y ***Significativo al $10 \%$

Fuente: Estimaciones propias 
El desempleo abierto en el municipio parece influir de manera positiva en el nivel de participación electoral del mismo, una vez que se corrige por el sesgo espacial; es decir, por cada punto porcentual en la tasa de desempleo, en promedio el nivel de participación electoral aumenta en 3.5\%. Por otro lado, un nivel alto de rezago social resultó tener un impacto en la participación electoral del 2012, comparado con lo que tendría el nivel bajo de rezago social. Por ejemplo, si un municipio tiene marginación alta y esta aumenta en $1 \%$, el incremento promedio en la participación electoral es de 0.0028 puntos superior al que tendría si fuera de marginación baja.

En todos los modelos el indicador de I de Moran de la regresión indica la presencia de autocorrelación espacial positiva y significativa. El modelo espacial que mejor ajusta el patrón de comportamiento de los datos para 2012 es el de Error Espacial, por lo cual, controlando por el sesgo espacial, se encuentra que la autocorrelación espacial es significativa para el coeficiente de lambda. Lo anterior implica que, por ejemplo, si el municipio tiene marginación alta, la participación electoral se eleva comparada con lo que aumentaría si fuera de marginación baja, siempre y cuando se controla el sesgo de autocorrelación espacial; de lo contrario, el aumento en el porcentaje de participación se subestimaría al reportar un impacto menor -de 0.0022 puntos-, tal y como lo indica el modelo $\mathrm{C}$. Cabe destacar que el nivel de correlación $\mathrm{R}^{2}$ de la regresión espacial mejora al llegar a un nivel de 0.5 , lo que indica que las variables explican mucho mejor cuando se controla por el sesgo espacial, comparado con el ajuste menor que reportan los otros modelos.

\section{Elecciones 2015}

Para explicar la variable dependiente del porcentaje de participación electoral (PPE) de las elecciones del 2015 a nivel municipal se tomaron como variables explicativas la tasa porcentual de desempleo del 2000, el nivel promedio de escolaridad del 2010 -en años-, la tasa de incidencia de delitos violentos por cada mil personas en 2011, el Índice Gini para 2015 y el índice de Rezago Social para 2000 clasificado en alto, media y baja. La tasa de desempleo del municipio parece influir de manera positiva en el nivel de participación electoral en 2015. Así, a mayor nivel de desempleo en el 2000, se refleja en una mayor participación de los ciudadanos. Sin embargo, la tasa de violencia resulta significativa y su impacto es negativo en la participación electoral cuando no se controla por la autocorrelación espacial. Ello que indicaría que, a una mayor tasa de violencia en el municipio, el nivel de participación se reduciría en 0.0001 puntos -modelo $\mathrm{C}$. 
TABLA 5 RESUltados 2015

\begin{tabular}{|c|c|c|c|c|c|c|c|}
\hline \multicolumn{8}{|c|}{ Variable dependiente: Participación electoral (\%) 2015} \\
\hline \multirow{2}{*}{$\begin{array}{l}\text { Variable } \\
\text { explicativa }\end{array}$} & \multicolumn{2}{|c|}{ Modelo A } & \multicolumn{2}{|c|}{ Modelo B } & \multicolumn{2}{|c|}{ Modelo C } & \multirow{2}{*}{$\begin{array}{c}\text { Modelo } \\
\text { D }\end{array}$} \\
\hline & Nivel & Semilog & Nivel & Semilog & Nivel & Semilog & \\
\hline $\begin{array}{l}\text { des- } \\
\text { emp2000 }\end{array}$ & $\begin{array}{l}* * 3.971 \\
(0.0354)\end{array}$ & $\begin{array}{r}* * \mathbf{0 . 0 5 6} \\
(0.0476)\end{array}$ & $\begin{array}{l}* * 4.220 \\
(0.0224)\end{array}$ & $\begin{array}{r}* * \mathbf{0 . 0 6 0} \\
(0.0320)\end{array}$ & $\begin{array}{r}* * \mathbf{3 . 7 6 4} \\
(0.0339)\end{array}$ & $\begin{array}{l}* * \mathbf{0 . 0 5 3} \\
(0.0459)\end{array}$ & $\begin{array}{r}* * * \mathbf{0 . 0 4 5} \\
(0.0534)\end{array}$ \\
\hline escol2010 & $\begin{array}{r}* \mathbf{- 3 . 7 0 2} \\
(0.0001)\end{array}$ & $\begin{array}{r}* \mathbf{- 0 . 0 5 6} \\
(0.0001)\end{array}$ & $\begin{array}{c}* * \mathbf{2} .448 \\
(0.0155)\end{array}$ & $\begin{array}{r}* * \mathbf{0 . 0 3 9 1} \\
(0.0105)\end{array}$ & & & \\
\hline tviol2011 & $\begin{array}{r}-0.401 \\
(0.3037)\end{array}$ & $\begin{array}{r}-0.005 \\
(0.4083)\end{array}$ & $\begin{array}{r}-0.064 \\
(0.8707)\end{array}$ & $\begin{array}{r}0.000 \\
(0.9701)\end{array}$ & $\begin{array}{r}* * \mathbf{- 0 . 7 7 6} \\
(0.0374)\end{array}$ & $\begin{array}{r}* * * \mathbf{0 . 0 1 0} \\
(0.0586)\end{array}$ & $\begin{array}{r}-0.008 \\
(0.1045)\end{array}$ \\
\hline Gini2015 & & & & & $\begin{array}{r}* \mathbf{1 0 9 . 6 6} \\
(0.00001) \\
\end{array}$ & $\begin{array}{r}* \mathbf{1 . 6 5 5} \\
(0.00001) \\
\end{array}$ & $\begin{array}{r}* \mathbf{1 . 2 9 0} \\
(0.0001) \\
\end{array}$ \\
\hline $\begin{array}{l}\text { d_maralta2 } \\
000\end{array}$ & & & $\begin{array}{r}* * \mathbf{1 0 . 5 7 7} \\
(0.0147)\end{array}$ & $\begin{array}{r}* * \mathbf{0 . 1 4 1} \\
(0.0297) \\
\end{array}$ & & & \\
\hline $\begin{array}{l}\text { d_marmed } \\
2000\end{array}$ & & & $\begin{array}{r}6.917 \\
(0.2609) \\
\end{array}$ & $\begin{array}{r}0.104 \\
(0.2621) \\
\end{array}$ & & & \\
\hline Constante & $\begin{array}{r}92.645 \\
(0) \\
\end{array}$ & $\begin{array}{r}4.583 \\
(0) \\
\end{array}$ & $\begin{array}{r}79.389 \\
(0) \\
\end{array}$ & $\begin{array}{r}4.402 \\
(0)\end{array}$ & $\begin{array}{r}22.494 \\
(0.0084)\end{array}$ & $\begin{array}{r}3.522 \\
(0)\end{array}$ & $\begin{array}{r}2.243 \\
(0.0001)\end{array}$ \\
\hline W_lnPPE15 & & & & & & & $\begin{array}{l}* * \mathbf{0 . 3 4 0} \\
(0.0164)\end{array}$ \\
\hline $\mathrm{N}$ & 51 & 51 & 51 & 51 & 51 & 51 & 51 \\
\hline $\mathcal{R}^{2}$ aiustada & 0.3715 & 0.3674 & 0.430116 & 0.470278 & 0.4402 & 0.4353 & 0.5342 \\
\hline $\begin{array}{l}\text { Aikake } \\
\text { criterion }\end{array}$ & 364.646 & -64.4937 & 361.432 & -66.3925 & 358.736 & -70.2861 & -73.5712 \\
\hline $\begin{array}{l}\text { Schwarz } \\
\text { criterion }\end{array}$ & 372.374 & -56.7664 & 373.023 & -54.8015 & 366.464 & -62.5588 & -63.9121 \\
\hline I de Moran & $* * 2.43$ & $* * 2.44$ & $* 2.60$ & $* 2.59$ & $* * 2.20$ & $* * 2.29$ & \\
\hline & $(0.0153)$ & $(0.0146)$ & $(0.0093)$ & $(0.0095)$ & $(0.0278)$ & $(0.0220)$ & \\
\hline
\end{tabular}

Se reporta para cada modelo los resultados a nivel de las unidades de la variables (NIVEL) y los resultados en términos de unidades logarítmicas en las variables independientes (Semilog).

Nota: entre paréntesis se reporta el p value

* Significativo al 1\%, **Significativo al 5\% y ***Significativo al $10 \%$

Fuente: Estimaciones propias

Dado que también el indicador de I de Moran señala evidencia de autocorrelación espacial en los datos, el modelo espacial que mejor explica estas relaciones es el de rezago espacial, llevando a un nivel de correlación de regresión de 0.53 . Así, controlando por autocorrelación espacial -modelo D- sigue conservando la violencia este impacto negativo, pero deja de ser significativo. Además, la variable de mayor impacto en la participación electoral en 2015 fue la desigualdad de ingresos, ya que un aumento del $1 \%$ en el índice de Gini-recordemos que este índice va en un rango de 0 a 1 - haría que la participación electoral en un municipio aumentara en 0.129 puntos, manteniendo igual el resto de las variables socioeconómicas. Esto significa que una elevada desigualdad de ingresos en la población -lo que podría implicar una menor cohesión social entre la población- 
hace que los ciudadanos participen más en las elecciones, buscando probablemente un cambio en su situación económica. Otra variable significativa es el desempleo, que reporta un impacto positivo; es decir, si aumenta la tasa de desempleo un $1 \%$, la participación electoral aumentaría en promedio 0.00045 puntos.

\section{Elecciones 2018}

Tabla 6. Resultados 2018

\begin{tabular}{|c|c|c|c|c|c|c|c|}
\hline \multicolumn{8}{|c|}{ Variable dependiente: Participación electoral (\%) 2018} \\
\hline \multirow{2}{*}{$\begin{array}{l}\text { Variable } \\
\text { explicativa }\end{array}$} & \multicolumn{2}{|c|}{ Modelo A } & \multicolumn{2}{|c|}{ Modelo B } & \multicolumn{2}{|c|}{ Modelo C } & \multirow{2}{*}{$\begin{array}{c}\text { Modelo D } \\
\text { Modelo } \\
\text { Error } \\
\text { Espacial }\end{array}$} \\
\hline & Nivel & Semilog & Nivel & $\begin{array}{c}\text { Semi- } \\
\log \end{array}$ & Nivel & Semilog & \\
\hline desemp2010 & $\begin{array}{r}-0.162 \\
(0.8686) \\
\end{array}$ & $\begin{array}{r}-0.002 \\
(0.9013) \\
\end{array}$ & $\begin{array}{r}0.451 \\
(0.6491) \\
\end{array}$ & $\begin{array}{r}0.008 \\
(0.6386) \\
\end{array}$ & $\begin{array}{r}0.193 \\
(0.8439) \\
\end{array}$ & $\begin{array}{r}0.002 \\
(0.8866) \\
\end{array}$ & $\begin{array}{r}-0.018 \\
(0.2039)\end{array}$ \\
\hline escol2015 & $\begin{array}{r}* 5.829 \\
(0) \\
\end{array}$ & $\begin{array}{r}*_{-\mathbf{0 . 0 9 7}} \\
(0) \\
\end{array}$ & & & $\begin{array}{r}*-4.734 \\
(0.0003) \\
\end{array}$ & $\begin{array}{r}*_{-\mathbf{0 . 0 8 3}} \\
(0.0002) \\
\end{array}$ & $\begin{array}{r}-0.033 \\
(0.2061) \\
\end{array}$ \\
\hline tviol2015 & $\begin{array}{r}* * *-1.461 \\
(0.0866)\end{array}$ & $\begin{array}{r}* * * \mathbf{0 . 0 2 7 6} \\
(0.051)\end{array}$ & $\begin{array}{r}-0.839 \\
(0.3288)\end{array}$ & $\begin{array}{r}-0.018 \\
(0.2212)\end{array}$ & $\begin{array}{r}-1.162 \\
(0.1689)\end{array}$ & $\begin{array}{r}* * *- \\
\mathbf{0 . 0 2 4} \\
(0.0953)\end{array}$ & $\begin{array}{r}* * \mathbf{- 0 . 0 2 7} \\
(0.0167)\end{array}$ \\
\hline Gini2015 & & & $\begin{array}{r}* 158.61 \\
3 \\
(0)\end{array}$ & $\begin{array}{r}* 2.576 \\
(0)\end{array}$ & & & \\
\hline $\begin{array}{l}\text { d_maralta200 } \\
0\end{array}$ & & & & & $\begin{array}{r}* * * 10.751 \\
(0.0512)\end{array}$ & $\begin{array}{r}0.135 \\
(0.1408)\end{array}$ & $\begin{array}{l}* * \mathbf{0 . 2 7 5} \\
(0.0219)\end{array}$ \\
\hline $\begin{array}{l}\text { d_marmed20 } \\
00\end{array}$ & & & & & $\begin{array}{r}2.970 \\
(0.7062) \\
\end{array}$ & $\begin{array}{r}0.051 \\
(0.6986) \\
\end{array}$ & $\begin{array}{r}0.069 \\
(0.5604) \\
\end{array}$ \\
\hline Constante & $\begin{array}{r}116.946 \\
(0)\end{array}$ & $\begin{array}{r}5.025 \\
(0)\end{array}$ & $\begin{array}{r}1.800 \\
(0.8965) \\
\end{array}$ & $\begin{array}{r}3.135 \\
(0)\end{array}$ & $\begin{array}{r}104.121 \\
(0)\end{array}$ & $\begin{array}{r}4.862 \\
(0)\end{array}$ & $\begin{array}{r}4.551 \\
0 \\
\end{array}$ \\
\hline Lambda & & & & & & & $\begin{array}{r}* \mathbf{0 . 5 8 1} \\
(0.00001)\end{array}$ \\
\hline $\mathrm{N}$ & 51 & 51 & 51 & 51 & 51 & 51 & 51 \\
\hline $\mathcal{R}^{2}$ aimstada & 0.38 & 0.39 & 0.39 & 0.38 & 0.41 & 0.40 & 0.57 \\
\hline $\begin{array}{l}\text { Aikake } \\
\text { criterion }\end{array}$ & 391.77 & -26.97 & 391.28 & -25.74 & 391.40 & -25.49 & -32.25 \\
\hline $\begin{array}{l}\text { Schwarz } \\
\text { criterion }\end{array}$ & 399.50 & -19.24 & 399.01 & -18.02 & 402.99 & -13.90 & -20.66 \\
\hline I de Moran & $\begin{array}{r}\mathbf{2 . 6 4} \\
(0.0083)\end{array}$ & $\begin{array}{r}* * \mathbf{2 . 1 7} \\
(0.0297)\end{array}$ & $\begin{array}{r}* 3.19 \\
(0.0015)\end{array}$ & $\begin{array}{r}\text { *3.16 } \\
(0.0016)\end{array}$ & $\begin{array}{r}\mathbf{3 . 1 3} \\
(0.0017)\end{array}$ & $\begin{array}{r}* \mathbf{2 . 6 1} \\
(0.0092)\end{array}$ & \\
\hline
\end{tabular}

Se reporta para cada modelo los resultados a nivel de las unidades de la variables (NIVEL) y los resultados en términos de unidades logarítmicas en las variables independientes (Semilog).

Nota: entre paréntesis se reporta el $\mathrm{p}$ value

* Significativo al 1\%, **Significativo al 5\% y ***Significativo al 10\%

Fuente: Estimaciones propias

Para explicar la variable dependiente del porcentaje de participación electoral (PPE) de las elecciones del 2018 a nivel municipal se tomaron como variables explicativas la tasa porcentual de desempleo del 2010, el nivel promedio de 
escolaridad del 2015 -en años-, la tasa de incidencia de delitos violentos por cada mil personas en 2015, el Índice Gini para 2015 y el índice de Rezago Social para 2000 clasificado en alto, medio y bajo nivel. La tasa de desempleo y el nivel de escolaridad dejan de ser importantes cuando se controla por autocorrelación espacial de los datos. En el modelo espacial, la tasa de violencia resulta significativa y su impacto es negativo en la participación electoral cuando se controla por la autocorrelación espacial, lo que indicaría que, por cada punto adicional en la tasa de violencia en el municipio, el nivel de participación se reduciría en promedio en 0.00027 puntos. Esto parece confirmar que la situación de violencia e inseguridad en algunos municipios afecta al deseo de la ciudadanía de participar en las elecciones, sobre todo para los comicios federales del 2018. El efecto de la marginación alta también resulta altamente significativo y favorece la participación de los ciudadanos en los comicios electorales.

Así observamos que el nivel de rezago social es la variable de mayor impacto en el modelo, ya que, si el municipio es de marginación alta en 2018, y si esta aumenta en un $1 \%$, la participación electoral se eleva en promedio 0.00274 puntos comparado con lo que sería si el municipio fuera de marginación baja.

\section{DISCUSIÓN}

En todo el análisis de regresión resultó evidente el problema de autocorrelación espacial de los datos; es decir, el nivel de participación electoral en un municipio determinado está relacionado con el nivel de participación electoral de su municipio cercano o vecino, así como también influyen los niveles de rezago, desigualdad, violencia o desempleo que puedan existir en los municipios cercanos o vecinos. Por este motivo, se corrige la estimación por el sesgo de dependencia espacial aplicando el modelo de rezago o error espacial. Este problema de la relación espacial ya había sido encontrado en otros casos aplicados a las preferencias electorales, o bien a la misma participación (Fiorino et al., 2016; Hernández-Hernández, 2015; Vilalta, 2008).

En general se encontró, mediante los modelos estimados, que los niveles de participación de los ciudadanos en los comicios en México de los años de 2009, 2012, 2015 y 2018 estuvieron determinados en su mayoría por variables tanto del entorno social como del económico.

Las variables socioeconómicas de mayor impacto en las cuatro elecciones fueron el desempleo, la tasa de incidencia de delitos violentos, el índice de rezago social y la desigualdad de ingresos. Especialmente, se encuentra que el 
rezago social alto es una variable que impacta más en el nivel de participación electoral de los municipios de Nuevo León, sobre todo en elecciones para la presidencia de la república de 2012 y 2018. En el caso de la relación positiva entre desempleo y participación electoral, esto ya se había encontrado en el estudio de Burden y Wichowsky (2012) a nivel entidad. El impacto en los resultados electorales y el grado de marginación también se había encontrado en los estudios de Vilalta (2008) y Hernández-Hernández (2015).

En 2012, al igual que lo observado para las elecciones de 2009, en que en los municipios con los niveles más elevados de carencias económicas son los que más participan, posiblemente alentados por los beneficios económicos y sociales que en tiempos electorales se observan en mayor intensidad por los partidos políticos. Ello que podría estar relacionado con lo que se conoce como clientelismo político. Un resultado similar se encuentra en Hernández-Hernández (2015).

La desigualdad de ingresos impactó solamente de manera significativa en las elecciones del 2015, lo que sugiere que a mayor desigualdad de ingresos municipal hay una mayor participación electoral. Adicionalmente, también es en 2018 donde por primera vez la violencia tiene un impacto negativo en la participación; es decir, a mayor violencia, menor participación electoral.

\section{CONCLUSIONES}

En este trabajo se ha analizado cómo la dependencia espacial interviene en el comportamiento de la participación electoral municipal en Nuevo León. Así, si un municipio específico predominan elevados niveles de desigualdad, bajos niveles de escolaridad, altos niveles de violencia y elevados niveles de índices de marginación, es probable que esto incida en la participación de los ciudadanos en los comicios electorales. En términos de la teoría de la estructuración, se puede indicar que las condiciones sociales están altamente relacionadas con la tesis de la participación electoral, por lo menos, con la evidencia encontrada específicamente para estos años.

Y si, a su vez, un municipio está rodeado de otros municipios con condiciones socioeconómicas similares, entonces existirá adicionalmente un efecto espacial en la participación de los ciudadanos en las elecciones. En este sentido, también se destaca y se justifica con estos resultados la literatura sobre sociología política que sugiere que en el análisis de la participación electoral intervienen, de forma categórica, las variables del entorno, pero también la espacialidad como factor estructurante a partir de la premisa de Giddens. 
Particularmente se comprueba la primera hipótesis planteada en la cual se establece que existe dependencia espacial de la participación electoral de la población municipal; es decir, municipios con altos niveles de participación electoral la incrementan en los municipios colindantes, mientras que municipios con bajos niveles de participación electoral la disminuyen la participación electoral en las municipalidades vecinas. A este fenómeno se le conoce como autocorrelación espacial. Es resultado del análisis del indicador de I de Moran que reportó un nivel positivo y significativo en relación a esta variable.

Para la segunda hipótesis se ha encontrado que, después de corregir con modelos de dependencia espacial, la presencia de autocorrelación espacial tiene una relación positiva con un mayor nivel de desigualdad económica, así como con un nivel alto de rezago social, y también con la participación electoral para un año determinado. Además, también existe una relación negativa entre los niveles de violencia y la participación electoral de un municipio. La violencia como fenómeno sociológico debe ser estudiado en todos sus efectos, pues si bien se puede señalar que el control del Estado sobre la misma ha ido paulatinamente a la baja -independientemente de que partido político gobierne-, es notable la evidencia de que en contextos de elecciones es un factor disuasivo, sobre todo que en zonas con alta presencia delictiva en donde ni siquiera se pudieron instalar casillas de votación.

También es importante destacar que si bien se ha señalado que Nuevo León, ubicado en frontera con Estados Unidos, es uno de los estados de mayor progreso económico del país, su comportamiento electoral no ha sido muy lejano del típico modelo de los estados con mayor grado de pobreza - principalmente en zona sur-sureste- en donde existe una alta correlación entre participación electoral y electores con desventajas económicas que son propensos a ser cooptados en la intención de su voto, como ha sido indicado en la literatura.

Un sugerente tema para futuras investigaciones es el análisis de los mecanismos corporativo-clientelares, que en el caso local pueden incentivar la compra del voto en zonas de alta marginación. Esto requiere otra estrategia de investigación - preferentemente de corte cualitativo- que no se ha planteado dentro de los objetivos de este trabajo. En otras palabras, se puede reconfigurar el análisis de la alta participación electoral en zonas marginadas por algún determinado partido político que incentive la participación electoral a cambio de prebendas monetarias o materiales de forma sostenida. 


\section{BIBLIOGRAFÍA}

Alarcón, V., \& García, N. (eds.). (2016). El proceso electoral del 2015. Instituciones, partidos y competencia. UAM-Iztapalapa; Tirant Lo Blanch.

Anselin, L. (1999). Spatial Econometrics. Bruton Center-School of Social Sciences, University of Texas.

Bartle, J., Birch, S., \& Skirmuntt, M. (2017). The local roots of the participation gap: Inequality and voter turnout. Electoral Studies, (48), 30-44. https://doi.org/10.1016/j.electstud.2017.05.004

Bedoya, J. F., Escobar, J.C., Sánchez, A. \& Nieto, F. (2019). Estudios sobre comportamiento electoral, ¿qué explica la participación en las urnas? Un estado del arte. Estudios Políticos, (54), 177-198. http://doi.org/10.17533/udea.espo.n54a09

Blais, A., \& Dobrzynska, A. (1998). Turnout in electoral democracies. European Journal of Political Research, (33), 239-261. https://doi.org/10.1023/A:1006802916256

Bobbio, N., Mattetuci, N., \& Pasquino, G. (2015). Diccionario de ciencia política. Siglo XXI.

Burden, B. C., \& Wichowsky, A. (2012). Unemployment and Voter Turnout. Documento de la reunión anual de APSA 2012. https://ssrn.com/abstract=2105212

Carreras, M., \& Castañeda-Angarita, N. (2019). Economic adversity and electoral participation of vulnerable socioeconomic groups. Electoral Studies, (57), 110-120. https://doi.org/10.1016/j.electstud.2018.11.004

Cebula, R., \& Alexander, G. (2017). Female Labor Force Participation and Voter Turnout: Evidence from the American Presidential Elections. Review of Economics and Institutions, $8(2), 1-29$. https://doi.org/10.5202/rei.v8i2.234

Chasco, C. (2003) Econometría espacial aplicada a la predicción-extrapolación de datos microterritoriales. Consejería de Economía e Innovación Tecnológica.

Comisión Estatal Electoral de Nuevo León. (2010). Memorias Estadísticas. Informe del Proceso Electoral Nuevo León 2008-2009. Comisión Estatal Electoral de Nuevo León.

Comisión Estatal Electoral de Nuevo León. (2013). Memorias Estadísticas. Informe del Proceso Electoral Nuevo León 2011-2012. Comisión Estatal Electoral de Nuevo León.

Comisión Estatal Electoral de Nuevo León. (2016). Memorias Estadísticas. Informe del Proceso Electoral Nuevo León 2014-2015. Comisión Estatal Electoral de Nuevo León.

Comisión Estatal Electoral de Nuevo León. (2019). Memorias y Estadísticas. Informe del Proceso Electoral y Consulta Popular Nuevo León 2017-2018. Comisión Estatal Electoral de Nuevo León.

Consejo Nacional de Evaluación de la Política de Desarrollo Social. (2015a). Medición del Índice de Gini. http://www.coneval.org.mx/Informes/Pobreza/Datos_abiertos/ODS/Indice_Gini_ingreso.csvg 
Consejo Nacional de Evaluación de la Política de Desarrollo Social. (2015b). Medición del Índice de Rezago Social. https://www.coneval.org.mx/Medicion/IRS/Paginas/Indice_Rezago_Social_2015.aspx

Cox, K. R. (1969). The Voting Decision in a Spatial Context. Progress in Geography, 1(1), 81-117.

Cutts, D., \& Fieldhouse, E. (2009). What small spatial scales are relevant as electoral contexts for individual voters? The importance of the household on the turnout at the 2001, general election. American Journal of Political Science, 53(3), 726-739. https://doi.org/10.1111/j.1540-5907.2009.00397.x

Elffers, H. (2003). Analysing neighbourhood influence in criminology. Statistica Neerlandica 57(3), 347-367. https://doi.org/10.1111/1467-9574.00235

Fiorino, N., Pontarollo, N., \& Ricciuti, R. (2016). Voter turnout in European Parliament elections: A spatial analysis. CESifo Working Paper Series 5910, CESifo. https://www.researchgate.net/publication/303785552_Voter_Turnout_in_European_Parliament_Elections_A_Spatial_Analysis

Giddens, A. (2006). La constitución de la sociedad. Bases para una teoría de la estructuración. Amorrortu.

Gilas, K. M. (2016). Entre el centralismo y el federalismo electoral. La nueva relación entre las autoridades electorales administrativas y jurisdiccionales a partir de la reforma 2014. En V. Alarcón \& N. García (eds.), El proceso electoral del 2015. Instituciones, partidos y competencia (pp. 53-72). UAM-Iztapalapa; Tirant Lo Blanch.

Gujarati, D. (2004). Basic Econometrics. The McGraw-Hill.

Hägerstrand, T. (1965). Aspects of the spatial structure of social communication and the difusión of information. Regional Science Association Papers XVI, Cracow Congress. https://link.springer.com/article/10.1007/BF01888934

Hernández, E. (ed.). (2010). Elecciones en tiempos de guerra. Baja California, Chihuahua, Durango, Nuevo León, Sinaloa, Tamaulipas, Veracruz. Universidad Autónoma de Sinaloa.

Hernández-Hernández, V. (2015). Análisis geoespacial de las elecciones presidenciales en México, 2012. EURE. Revista Latinoamericana de Estudios Urbanos Regionales 41(122),185207.

Huckfeldt, R., \& Sprague, J. (1995). Citizens, Politics and Social Communication: Information and Influence in an Election Campaign. Cambridge University Press.

Instituto Nacional de Geografía y Estadística. (2000). Censo de Población y Vivienda 2000. https://www.inegi.org.mx/programas/ccpv/2000/

Instituto Nacional de Geografía y Estadística. (2010). Censo de Población y Vivienda 2010. https://www.inegi.org.mx/programas/ccpv/2010/

Lazarsfeld, P., Berelson, B., \& Gaudet, H. (1960). El pueblo elige. Estudio del proceso de formación del voto durante una campaña presidencial. Ediciones 3. 
Lavezzolo, S. (2008). Adversidad económica y participación electoral en América Latina 19802000. Revista Española de Ciencia Política, (18), 67-93.

León, L. E. (2019). Perfil ideológico e intención de voto unidimensional. En A. Díaz \& C. J. Vásquez (eds.), Perfiles del electorado nuevoleonés (pp. 67-93). Comisión Estatal Electoral de Nuevo León.

Lipset, S. M. (2001). Algunos requisitos sociales de la democracia: desarrollo económico y legitimidad política. En A. Batlle i Rubio (ed.), Diez textos básicos de ciencia política (pp. 113-150). Ariel.

Lois, M. (2013). Lugar y política: ¿una lógica geográfica del comportamiento electoral? Revista Española de Ciencia Política, (25), 97-106.

Losada, R., \& Casas, A. (2008). Enfoques para el análisis político. Historia, epistemología y perspectivas de la ciencia política. Pontificia Universidad Javeriana.

Manski, C. (1993). Identification of Endogenous Social Effects: The Reflection Problem. Review of Economic Studies, 60(3), 531-542.

Marván, M. (2014). La reforma electoral de 2013-2014. Centralización como alternativa al sospechosismo. Revista Análisis Plural, $1^{\mathrm{er}}$ semestre de 2014. https://rei.iteso.mx/handle/11117/1461

Mazurek, H. (2009). Espacio y territorio. Instrumentos metodológicos de investigación social [Versión EPUB]. IRD Editions.

Medellín, L., \& Murillo, J. (2014). El sistema político electoral en Nuevo León. Elecciones y efectos en el sistema de partidos politicos 1979-2012. Facultad de Derecho y Criminología-UANL; Tribunal Electoral del Estado de Nuevo León; Asociación Mexicana de Ciencia Política.

Medellín, L., \& Ontiveros, C. (2013). Elecciones comparadas en Nuevo León y Sinaloa en un contexto de crimen organizado. Ciencia UANL, 16(63), 55-63.

Montecinos, E. (2007). Análisis del comportamiento electoral: De la elección racional a la teoría de redes. Revista de Ciencias Sociales, 13(1), 9-22.

Montero, J. C. (2019). Determinantes del voto del elector nuevoleonés. En A. Díaz \& C. J. Vásquez (eds.), Perfiles del electorado nuevoleonés (pp. 34-62). Comisión Estatal Electoral de Nuevo León.

Moreno, R., \& Vayá, E. (2002). Econometría especial: nuevas técnicas para el análisis regional. Una aplicación a las regiones europeas. Investigaciones Regionales, (1), 83-106.

Muschick, M. (2004). Teorías del comportamiento electoral y algunas de sus aplicaciones. Revista Mexicana de Ciencias Políticas y Sociales, 46(190), 47-70.

O' Loughlin, J. (2001). Geography and democracy: the spatial difusión of political and civil right. In G. Dijkink. \& H. Knippenberg (eds.), The territorial factor. Political geography in a globalising world (pp. 77-96). Vossiuspers UvA. 
O'Loughlin, J. (2003). Spatial analysis in political geography. In J. Agnew, M. Katharyne \& G. Toal (eds.), A companion to political geography. (pp. 30-47) Blackwell Publishing Company.

Pacheco, G. (2000). Caleidoscopio electoral: Elecciones en México, 1979-1997. IFE-UAM-FCE.

Redfield, R. (1965). Peasant society and culture. University of Chicago Press.

Rosenstone, S. J. (1982). Economic Adversity and Voter Turnout. American Journal of Political Science, 26(1), 25-46. https://doi:10.2307/2110837

Rostow, W. W. (1960). Las etapas del crecimiento económico. Un manifiesto no-comunista. Fondo de Cultura Económica.

Secretariado Ejecutivo del Sistema Nacional de Seguridad Pública. (2019). Estadísticas de incidencia delictiva municipal 2011-2017. https://www.gob.mx/sesnsp/acciones-y-programas/incidencia-delictiva-del-fuero-comun?idiom=es

Sonnleitner, W. (2007). Participación electoral y desarrollo humano: apuntes metodológicos para el análisis territorial y multidimensional del voto en México y Centroamérica. Estudios Sociológicos, 25(75), 813-835.

Sonnleitner, W. (2017). Variedades del voto: hacia una sociología plural del sufragio particular. Estudios Sociológicos, 35(104), 429-448. https://doi:10.24201/ES.2017V35N104.1536

Soto, I., \& Cortez, W. (2014). Determinantes de la participación electoral en México. Estudios Sociológicos, 32(95), 323-353.

Valdez Zepeda, A., \& Paniagua Vázquez, A. (2011). Criminalidad, inseguridad pública y comportamiento de los electores: un análisis del proceso electoral estatal 2010 en Ciudad Juárez, Chihuahua. Estudios fronterizos, 12(23), 49-78.

Vilalta, C. (2003). Perspectivas geográficas en la sociología urbana: la difusión espacial de las preferencias electorales y la importancia del contexto local. Estudios Demográficos y Urbanos, 18(3), 537-557. https://doi.org/10.24201/edu.v18i3.1158

Vilalta, C. (2008). ¿Se pueden predecir geográficamente los resultados electorales? Una aplicación del análisis de clusters y outliers espaciales. Estudios Demográficos y Urbanos, 23(3), 571-613. https://doi.org/10.24201/edu.v23i3.1322

Weaver, R. (2014). Contextual influences of political behavior in cities: toward urban electoral geography. Geography Compass, 8(12), 874-891. https://doi.org/10.1111/gec3.12186

Zineterer, T., \& Nohlen, D. (2006). Participación electoral. En D. Nohlen (ed.), Diccionario de ciencia política. Teoría, métodos, conceptos (Tomo II, pp. 1002-1004). Editorial Porrúa; El Colegio de Veracruz. 


\section{ANEXO 1}

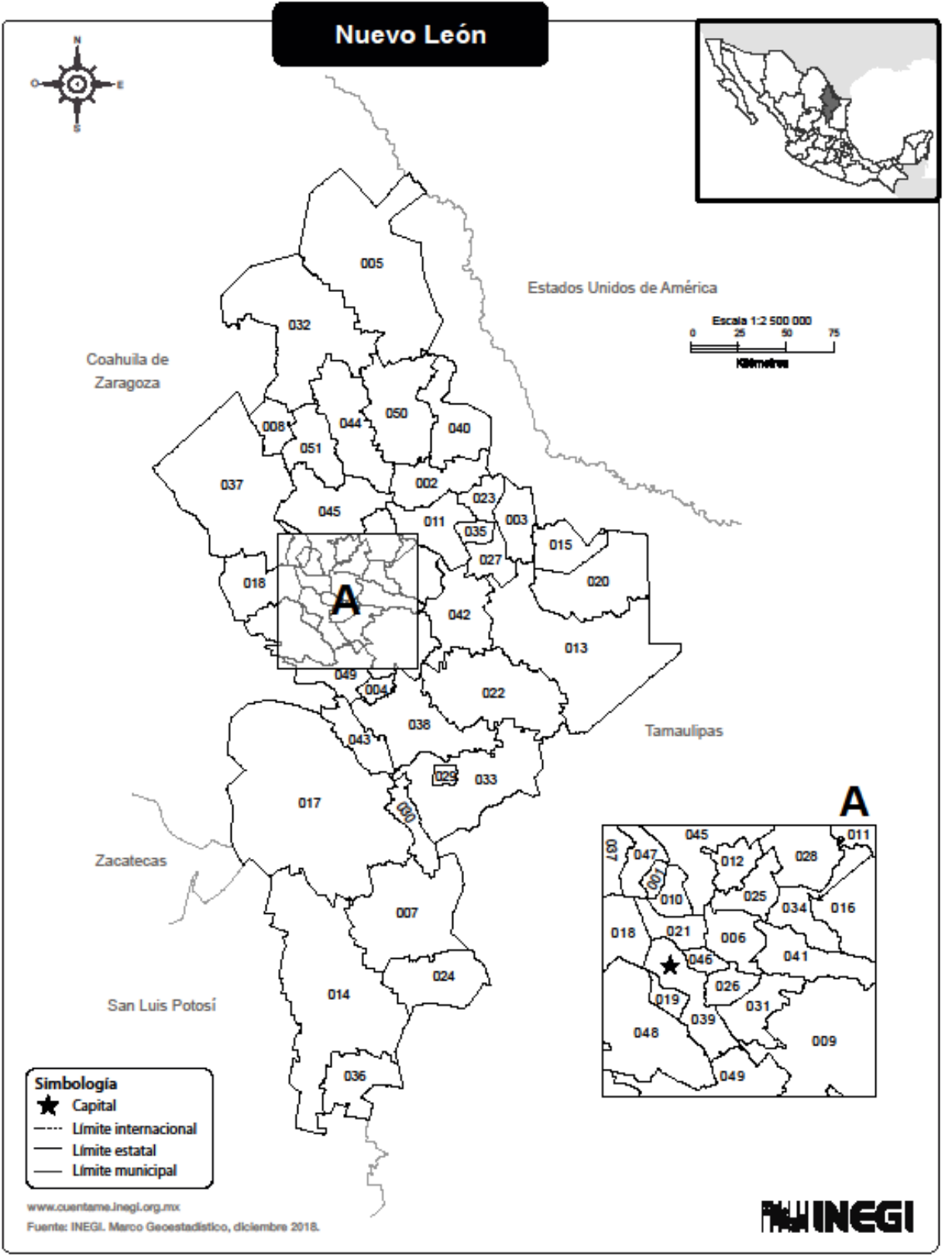




\section{Municipios:}

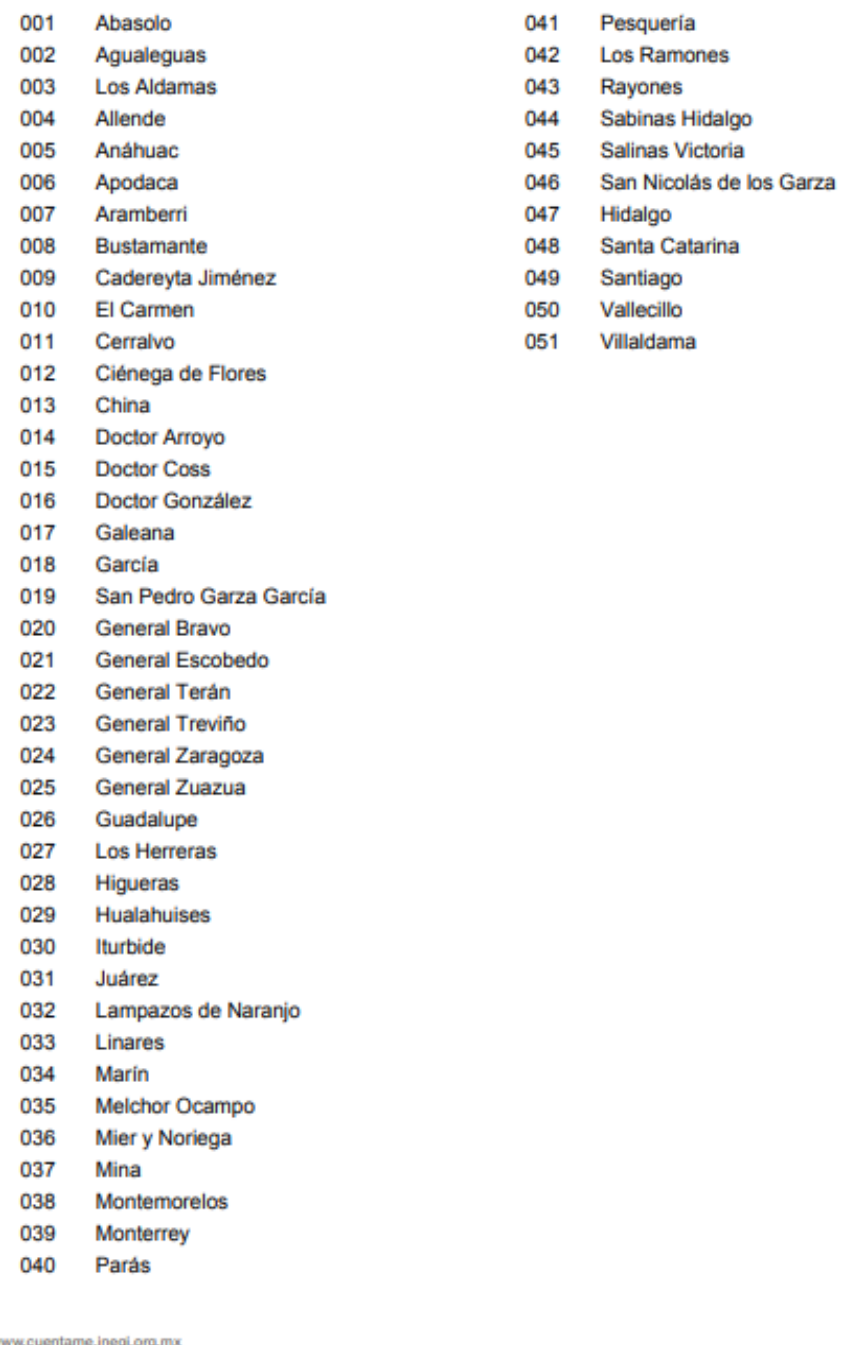


